\title{
Spectral Density Functions for Cisordered Systems
}

Dennis Gene Hall

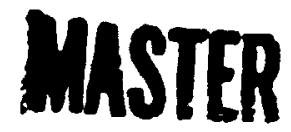




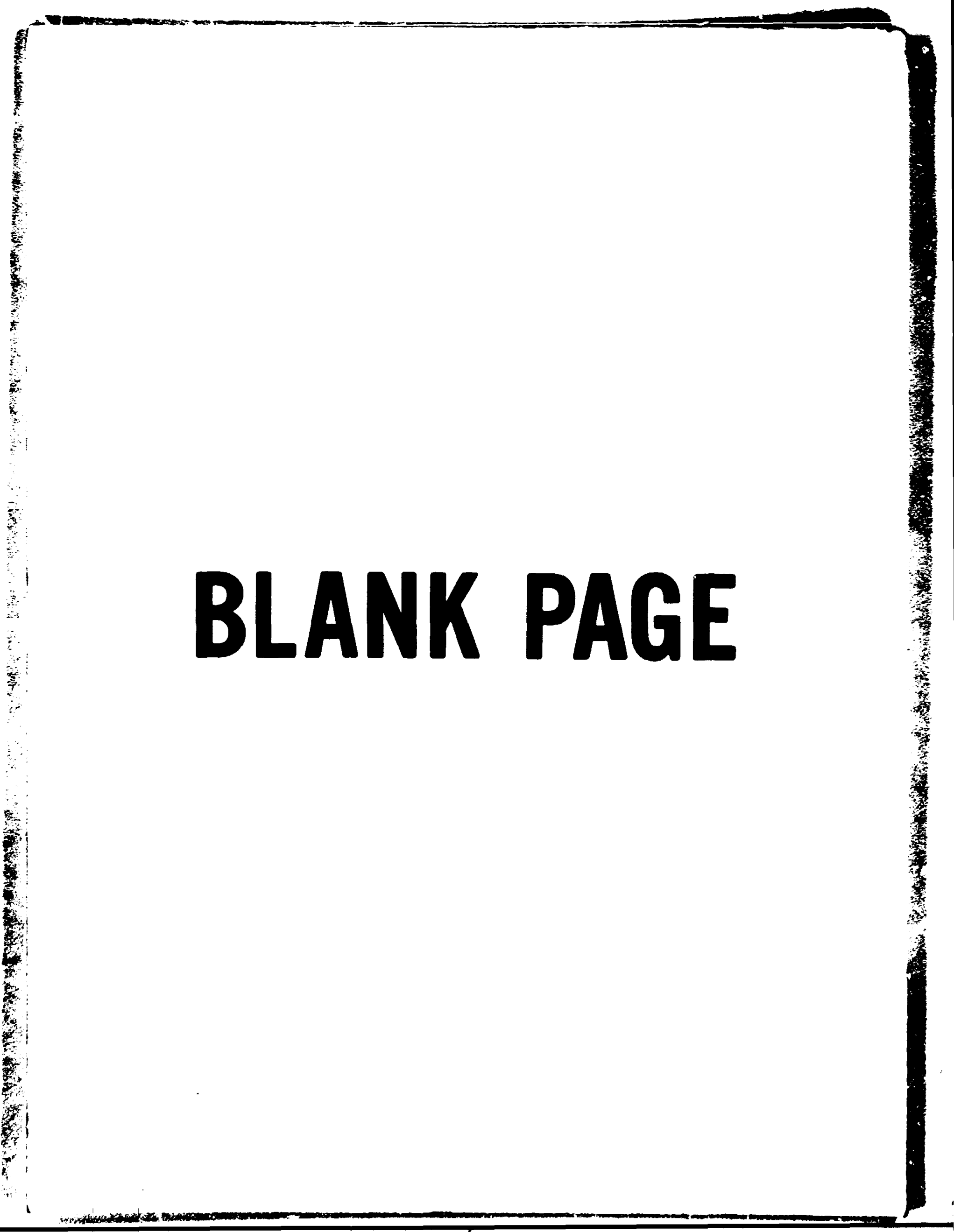


Printed in the United States of America. Avaitale from National Technical Information Sevice

U.S. Depertment of Commerce

5205 Port Roval Rasd, Springfield, Virgimia 22161

Price: Prinad Copy Sere. Mierofiche $\$ 2.25$ 5. 2

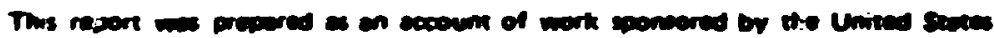

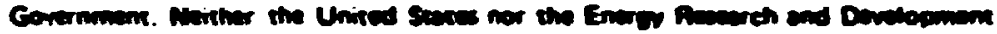

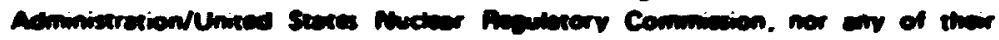

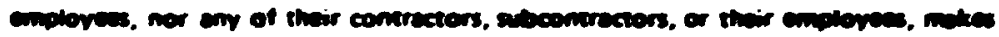

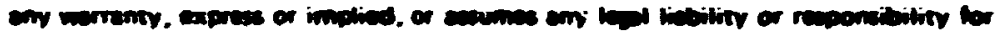

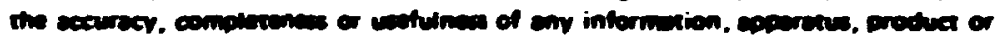

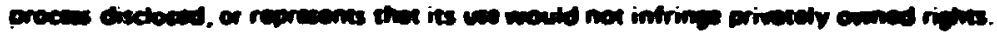


Oank-5184

Distribution

Category uc-25

Contract No. W-7405-eng-26

METALS AND CERAMICS DIVISION

SPECTRAL DENSITY FUNCTIONS FOR DISORDERED SYSTENS

Dennis Gene Hall

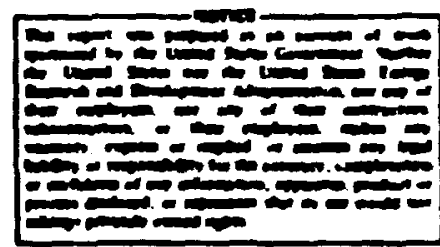

A dissertation presented to the Graduate Council of The University of Tennessee in partial fulfillment of the requirements for the degree of Doctor of Philosophy.

Dowe Published: November 1976

OAK RIDGE MATIONAL LABORATORY

Oak Ridge, Tennessee 37830

operated by

UNION CARBIDE CORPORATION

for the

ENERGY RESEARCH AND DEVELOPMENT ADMINISTRATION 


\section{ACKROULEDCATH-S}

The author would like to thank $2 \cdots, y$. S. Faulkner of the Oak Ridge :tational Laboratory $50:$ sunge ssting this work and for his continued interest ant sis-? of the research, and Drs. W. H. Butler and J.J. Olson for many helpful discussions. The aurhcr is grateful to the staff of the Metals and Ceramics livision of the Oak Ridge Mational Laboratory operated by Union Carbice Corporation for the Energy Research and Developmen: Natainistration for their hospitality during the performance of t:as mork. Thanks are extended to the Physics Department at the :iniversity of Tennessee for providing financial suf:ort in the form cf teaching assistantships and to the Unitsd Stutes Energy Research and Development Administration fs:t providing aupport in the form of a Laboratory Fellowshif. 


\section{ABSTARCT}

This work describes a Monte carlo calculation of the spectral density function $A(k, E)$ foi a one-dimensional mode: of an anorphous solid. The nodel contains an adjustable short-range order parameter and $A(K, E)$ is obtained for several values of that parame:s:. This Maniltonians considered are: (I) the hamiltonian describing an electron woving in a potential consisting of randonly placed delta functions, (2) the Hawiltonian describing a system of coupled harmonic oscillators, and (3) a tight-binding Hamiltonian describing in a simplified way either electrons or spin-waves.

A major ain of the present work was to apply the insight gained from this numprical calculation to interpret the qualitative features of recent neutron scattering data on amorphous systems. In the absence of a reliable theory of elewentary excitations in structurally disordered systems. model calculations becone extremely important in pointing out the properties to be expected of such systems. The spectral density $A(k, E)$ is a particularly important quantity in this regard since $A(k, E)$ gives the single-particle contribution to the dynamic form factor of neutron scattering theory. 
As another application of the ndel calculation, the spectral density is obtained is the quasicrystalline approximation (QCA) for the problem of an electron in a one-dimensional liquid meta!. The och and exact spectral densities are rmpared and it is shown that the OCA fails in the strong scattering regime. 
TABLE OF CONTENTS

CHAPTER

PAGr:

I. INTRODUCTION . . . . . . . . . . . . . 1

Substitutional Disorder: The Binary Alloy . . 3

Structural Disorder . . . . . . . . . . . 10

Numerical Investigatic.s . . . . . . . . . 19

II. THE SPECTRAL DENSITY FOR AN ELCCTRON IN $A$

ONE-DIFLASYONAL LIQUID METAL . . . . . . . . 23

The Model Systen . . . . . . . . . . . 23

Calculation of the Spectral Density . . . . . 26

Convergence of $\mathbf{A}(\boldsymbol{k}, \mathrm{E})$. . . . . . . . . . . . 34

Comparison of tre $Q \mathrm{CA}$ and Exact Resuits . . - 10

III. TIGHT-BINDING SYSTEMS: THE EFFFCTS OF

SHORT-RANGE ORDER ................. 49

The Spectral Density............. 49

Results of the Calculation . . . . . . . . 55

Felation to Experiment . . . . . . . . . . 66

IV. SUMMARY ..................... 73

LIST OF REFERENCES - . . . . . . . . . . . . . 77

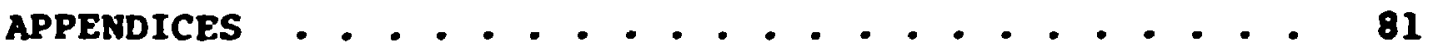




\section{LISt OF FIGURes}

FIGURE

PAGF

1. Schematic Representation of the Bard-Rod

Liquid . . . . . . . . . . . . . . . . 55

2. Comparison of OCA (Smooth Curve) and Fxact

(Histograa) Spectral Densities $A(k, E)$ for

$a=.50$ (Foreground) and $a=.91$

(Background) and Energy $E=.50$

3. Comparison of QCA (Smooth Curve: and Exact

(Histcgraa) Spectral Densities $A(K, F)$ for

$a \leq .31$ and Energy $E=5.0 . . . . . . . . .$.

4. Exact Spectzal Density $A(k, E)$ for $a=.91$

and $E=-1.0$...................

5. Spectral Density $\mathbf{h}(Q, w)$ as a function of $Q$ for $\omega=.50$ and $a=.75$ for the vibrational

System . . . . . . . . . . . . . . . .

6. Spectral Density $\mathbf{A}(\mathbf{Q}, \mathrm{E})$ as a Function of $Q$ for $a=.85$ and Four values of $E$ for the Tight-

Binding System . . . . . . . . . . . . .

7. Spectral Density $\mathbf{A}(Q, E)$ as a Pusction of $Q$ for $a=.75$ and Four Values of $E$ for the Tight-

Binding System . . . . . . . . . . . . . .

8. Spectral Density $\mathbf{A}(Q, E)$ as a Function ct $Q$ for $E=.50$ and Three Values of $a$ for the Tight-

Binding Systen . . . . . . . . . . . . .

9. Spectral Density $A(Q, \omega)$ as a Punction of $Q$ for $\alpha=.85$ and Four Values of $\omega$ for the

Vibrational systen . . . . . . . . . . . .

10. Spectral Density $A(Q, \omega)$ as a Punction $r t Q$ for $a=.75$ and Pour Values of $\omega$ for the

Vibrational system . . . . . . . . . . . . .

11. Spectral Density $A(Q, w)$ as a Punction of $Q$ for $\omega=.10$ and Three Values of a for the Vibrational system . 
FIGURE

PAG:

22. Spectral Density $\mathbf{A}(0, w)$ as a Punction of $Q$ Sor $\omega=.50$ and Three Values of a for the Vibrational syster . . . . . . . . . . . . . 64

13. Meutron scattering Intensity as a Punction of $Q$ for Severai Energies (Bnergy Increases Verticall; fron rook et al. (30) . . . . . . 67

14. Suggested Analysis of Data in Figure 13; from Hook et al. (30) .................. 


\section{CHAPTER I}

\section{INTRODUCTION}

Throughout the history of Solid State Physics, the ordered arystal has played the central role in both theoretical and experimental investiga:ions. There is, however, a growing interest in systems such as liquid wetals and anorphous solids for which disorder is a fundanental conceot. Disorder can be categorized as substitutional, structural, or a combination of the two. Substitutional disorder is characteristic of the binary alloy $A_{x} B_{1-x}$ in which $A$-atoms and B-atoms are randomly distributed on the sites of a periodic lattice. Even in a perfectly random system there will be ciusters of $A$-atons, clusters of B-atoms, and so on, but these tendencies can be accentuated if short-range order exists. Structural dis.rder is typical of an amorphous solid or a liquid wetal in which the atomic sites aze randomly distributed. Local correlations of the atomic positions must be present at least to the extent necessary to prevent overlap of the atomic sites. In attempting to predict the properties of the electronic excitations in disordered systems, the inherent lack of symmetry presents a formidable theoretical problem. A silitable approximation which adequately describes the electronic properties of substitutional binary alloys in the 
absence of short-range order, the coherent potential approximaticn, was found in 1967. No approximation has yet proven successful in describing the properties of systems with structural disorder where the inclusion of the effects of short-range order is essential.

The present work describes a numerical investigation of the properties of elementary excitations in a one-dimensional model of a structurally disordered system. The spectral density function $A(k, E)$, related to the imaginary part of the single-particle Green' $\_$function, has been calculated for several Hamiltonians. $A(K, E)$ describes the probability of finding a single-particle excitation with energy $\mathbf{E}$ and wavenumber $k$. This $k$-dependence makes the spectral density particularly sensitive to the effects of structural disorder. In the absence of a reliable theory for excitations in such systems as amorphous solids and liquid metals, numerical investigations become the only means available for interpreting experimental results. It is the principal aim of this work to apply the insight gained from the model calculations to recent neutron inelastic scattering measurements of the magnetic excitations in an amorphous ferromagnet. The calculations in Chapter III lead to a reasonable conclusion regarding these magnetic excitations as well as a prediction of the properties of vibrational excitations in an amorphous solid. The second aim of this 
work is a comparison of the exact results with $A(k, E)$ obtained in the quasicrystalline roximation (QCA). This comparison points out the failure of the ... n the strong-scattering regime and is described in Cha.... i.

This chapter will describe " efforts in the theory of electronic excitation. Isordered systems. Part A will be devoted to a discussion of the coherentpotential approximation for binary alloys. Part B will demonstrate how structural disorder is incorporated into theories of electronic excitations in liquid metals. Part $C$ will consider numerical investigations.

\section{A. Substitutional Disorder: The Binar: Alloy}

Hociern theories of the electronic properties of disordered systems are generally formulated in terms of Green's functions. The Green's function $G^{ \pm}(\vec{r}, \vec{r} \cdot ; E)$ is a solution of the equation

$$
(E-H \mp \text { in }) G^{ \pm}\left(\vec{r}, \vec{r}^{\prime} ; E\right)=\delta\left(\vec{r}-\overrightarrow{\mathbf{r}}^{\prime}\right), \quad I . A . I
$$

where $H$ is the Hamiltoniar:

$$
H=-\frac{h^{2}}{2 m} \nabla^{2}+\sum_{n=1}^{N} v_{n}\left(\vec{r}-\vec{R}_{n}\right), \quad I . A .2
$$

with $V_{n}$ the potential for the fon at site $n$ and $n$ is a 
positive infinitesimal. Upon introducing the exact eigenstates $\psi_{n}(\vec{r})$ of the Hamiltonian with eigenvaikes $E_{n}$. $G^{ \pm}(\vec{r}, \vec{r} \cdot ; E)$ can be written

$$
G^{ \pm}(\vec{r}, \vec{r} \cdot ; E)=\sum_{n} \frac{\psi_{n}(\vec{E}) \psi_{n}(\vec{r} \cdot)}{E-E_{n} \bar{\ddagger} \text { in }} . \quad I . A .3
$$

It is more convenient to work with the propagator, or resolvent,

$$
G(2)=(2-H)^{-1} \text {. }
$$

defined such that

$$
G^{ \pm}(\vec{r}, \vec{r} \cdot ; E)=\langle\vec{r} ; G(z) \mid \vec{r} \cdot\rangle
$$

with $z=E \mp$ in. Both the Green's function and the resolvent defined above contain explicit dependence upon the arrangement of atoms in a particular system. The quantity of interest is the configuration averaged Green's function, or the configuration averaged resolvent, denoted by $\left(G^{ \pm}\left(\vec{r}, \vec{r}^{\prime} ; E\right)\right)$, or $(G(2))$, where 6 .. d denotes an average over all configurations in the statistical ensemble.

A history of the developments in the theory of the electronic properties of binary alloys has been given by Faulkner (1) and will not be repeated here. The most successful approximation used in the alloy problem has been 
the coherent potential approximation (CPA) of Soven (2). The CPA has been discussed by Velicky, Rirkpatrick, and Ehrenreich (3) and has been successfully applied to real systees by stocks, Williams, and Faulkner (4, 5,6). In attempting to calculate $\langle G(x)\rangle$ it is assumed that there exists an effective Hamiltonian $H_{\text {eff }}$ such that

$$
G(z)\rangle=\left(z-H_{\text {eff }}\right)^{-1} \text {. }
$$

with $\mathrm{H}_{\text {eff }}$ an unknown quantity. Let $\mathrm{K}$ be some starting approximation to $H_{\text {eff }}$. The propagator associated with $K$ is th: $=\mathbf{n}$

$$
R(z)=(2-K)^{-1}
$$

Using the operator identity

$$
(A-B)^{-1}=A^{-1}+A^{-1} B(A-B)^{-1} \text {. }
$$

with $A=2-K$ and $B=H_{\text {eff }}-K$ gives

$$
\left.\langle G(z)\rangle=R(z)+R(z)\left(H_{\text {eff }}-K\right): G(z)\right\rangle \cdot I . A .9
$$

Similarly, Eq. I.A.8 with $A=2-K$ and $B=K-K$ gives

$$
G(z)=R(z)+R(z)(H-K) G(z) \text {. }
$$

Defining the T-matrix for the system such that

$$
T(K) R(z)=(H-K) G(z) \text {. }
$$


one can write

$$
G(z)=R(z)+R(z) T(K) R(z) .
$$

with $T(K)$ satisfying the equation

$$
T(K)=(H-K)+(H-K) R(z) T(K) .
$$

Eq. I.A.12 can be used to establish a relationship between $G(z)$; and $\langle T(K)$;

$$
\langle G(z)\rangle=R(z)+R(z)\langle T(R)\rangle R(z)
$$

Combining Eqs. I.A.6, I.A.7, and I.A.14 gives

$$
H_{\text {eff }}=K+\langle T(K)\rangle\left\{1+R(z)\langle T(K)\rangle j^{-1}, \quad I . A .15\right.
$$

This equation demonstrates that the self-consisten solution $H_{\text {eff }}=\mathbf{K}$ would be obtained if $K$ were defined such that

$$
\langle\mathbf{T}(\mathbf{K})\rangle=0
$$

No approximations have been made up to this point. However, Eq. I.A.16 is not particularly helpful as the T-matrix for the complete system is not known.

In an effort to relat.e the condition expressed in Eq. I.A.16 to single-site operators, let us make the assumption that $(H-K)$ can be written as a sum of the site quantities $v_{n}\left(\vec{r}-\vec{R}_{n}\right)=v_{n}$. That is, assume 


$$
\begin{gathered}
7 \\
H-K=\left\{V_{n} .\right.
\end{gathered}
$$

Combining this assumption with Eq. I.A.11 gives the equation

$$
\sum_{n} V_{n} G(z)=T(K) R(z) \text {. }
$$

or, with Eq. I.A.12,

$$
T(R)=\sum_{n}\{1+R(z) T(K)\}
$$

Introducing the quantities $Q_{n}(x)$ and $t_{n}(x)$ such that

$$
T(K)=\sum_{n} Q_{n}(K) \text {, }
$$

and

$$
t_{n}(K)=\left\{1+v_{n} R(2)\right\}^{-1} v_{n} \text {. }
$$

the single-scattering atrix for the potenial $v_{n}$ (note that $t_{n}$ obeys the relation $t_{n}=v_{n}+v_{n} R(z) t_{n}$, a result which will be used later) an expression can be developed relating $T(X)$ tc contributions from single-sites. Setting

$$
\begin{aligned}
Q_{n}(x) & =v_{n}\{1+R(z) T(x)\} \\
& =v_{n}\left\{1+R(z) Q_{n}(x)+R(z)=n Q_{m}(x) ;, I . A .22\right.
\end{aligned}
$$

and rearranging terms yields

$$
T(x)=\sum_{n}(x)\left\{1+R(z)=\sum_{n} c_{m}(x)\right\} .
$$


Iteration of the above equation results in the series

$$
\begin{aligned}
T(x)= & \sum_{n} t_{n}(x)+\sum_{n} t_{n}(x) R(z)=\sum_{n} t_{i}(x) \\
& +\sum_{n} t_{n}(x) R(z)=\sum_{n} t_{n}(x) R(z), \sum_{n} t_{l}(x)+\ldots
\end{aligned}
$$

I.A. 24

as first given by Eawards (7).

At this point, the approximation is made that the average of the product in Eq. I.A.23 way be replaced by the product of the averages,

$$
T(K)=\sum_{n}\left\langle t_{n}(K)\right\rangle\left\{1+R(z) m \sum_{n}\left\langle Q_{m}(K)\right\rangle\right\} . \quad I . A .25
$$

The terms neglected in writing Eq. I.A.25 are

$$
\left\langle t_{n}(K) R(2) \sum_{n}\left\{Q_{m}(K)-\left\langle Q_{m}(K)\right\rangle\right\}\right\rangle, \quad I . A .26
$$

and inciude the statistical correlations between site $n$ and all other sites. In Eq. I.A.25, the wave incident on site $n$ contains no information about the actual configuration surrounding sitn $n$. By noting that $T-Q_{n}=m \sum_{n} Q_{m}$ ' Eq. I.A. 25 becomes

$$
\langle T(K)\rangle=\xi_{n}\left\{1+\left\langle t_{n}(K)\right\rangle R(z)\right\}^{-1}\left\langle t_{n}(K)\right\rangle\{1+R(z)\langle T(K)\rangle\},
$$

and inserting this into Eq. I.A.15 givas the re,ult 


$$
H_{\text {eff }}=K+\sum_{h}\left(l_{n}(K)\right)\left(1+R(z)\left\langle t_{n}(K)\right\}\right\}^{-1} .
$$

Within the approximation that correlations between scatterers can be neglected, the self-consistent solution $B_{\text {eff }}=K$ is obtained by determining $x$ from the equation

$$
\left\langle t_{n}(R)\right\rangle=0 .
$$

For a binary alloy, the site $n$ is occupied by an A-atom or a B-atom and the condition expressed in Eq. I.A.29 becomes

$$
c_{A} t_{A}(K)+c_{B} t_{B}(R)=0 \text {, }
$$

where $c_{A}$ and $c_{B}$ are the (known) concentrations of $A$-atoms and $B$-atoms, respectively. After solving for $K$, which may not be a simple task, $R(z)$ can be formed and is now equal to $\langle G(z)\rangle$. Prom $\langle G(z)\rangle$, the density of states $\langle P(E)\rangle$ and related quantities are obtained. For example,

$$
\langle D(E)\rangle=-\frac{1}{\pi} \operatorname{ImTx}\langle G(z)\rangle \text {. }
$$

where the abbreviation $\operatorname{Tr}$ indicates the trace of a matrix. The CPA is a single-site approximation. The physical content of the approximation lies in the replacement of the actual disordered syste' by an effective medium. The medium is characterized by a coherent potential which does not depend upon the atoric sites or what occupies those sites, 
but does depend upon the energy. The medium is determined from the condition that if that part of the coherent potential associated with the site $n$ is renoved and replaced by an actual constituent of the system (an A-atom or a B-atom) there should be no additional scattering introuced on the average. The CPA is a self-consistent approximation in the sense that the resolvent describing propagation of a wave in the effective medium is the actual $\langle G(z)\rangle$ which itself depends upon the medium in question.

Efforts have been made by several authors to go royond the single-site approximation to describe scattering by pairs and higher configurations. A recent numerical investigation by Nickel and Butler (8) has demonstrated the existence of nonanalyticities in $\langle G(2)\rangle$ calculated from various multi-site schemes. They suggest that nonanalyticity may be a general feature of higher-order self-consistent approximations and such approximations should be treated with caution until their analytic properties are fully appreciated.

\section{B. Structural Disorder}

The problem of describing the properties of electronic excitations in structurally d.sordered solids and liquil metals is considerably different from the alloy problem described above. It is necessary to include at sone stage 
of the calculation an appropriate description of the statistical correlations of the scattering centers. The paragraphs which follow provide a synopsis of several theoretical attempts at incorporating short-range order into a theory of the electronic properties of a liquid metal. The Hamilzonian under consideration is of the same form as that given in Eq. I.A.2 and for convenience let $H_{0}=\frac{n^{2}}{2 m} \nabla^{2}$. Defining $G(z)=(2-H)^{-1}$ and $G_{0}(z)=\left(2-H_{0}\right)^{-1}$ one can write the average propagator $\langle G(z)$; in terms of an average $T$-matrix

$$
\langle G(z)\rangle=G_{0}(z)+G_{0}(z)+G_{0}(z), \quad I . B .1
$$

as in Eq. I.A.14. The notation $\langle\mathrm{T}\rangle$ means

$$
\langle T\rangle=\int T\left(\vec{R}_{1}, \vec{R}_{2} \ldots, \vec{R}_{N}\right) P\left(\vec{R}_{1}, \vec{R}_{2}, \ldots, \vec{R}_{N}\right) d^{3} R_{1} d^{3} R_{2} \ldots d^{3} R_{N}, I . B .2
$$

where $P\left(\vec{R}_{1}, \vec{R}_{2}, \ldots, \vec{R}_{N}\right) d^{3} R_{1} d^{3} R_{2} \ldots d^{3} R_{N}$ gives the probability that $N$ ions will be centered at $\vec{R}_{1}, \vec{R}_{2} \ldots, \vec{R}_{N}$ within $d^{3} R_{1} d^{3} R_{2}$ $\ldots . \mathrm{d}^{3} \mathrm{R}$ - In Eq. I.B.2, the dependence of the T-matrix upon the ion positions has been made explicit. As in Eq. I.A.20, the T-matrix is written as a sum of single-site contributions $T=\sum_{\alpha} Q_{\alpha} \cdot$ Eq. I.B.2 becomes

$$
\langle T\rangle=\sum_{a} s Q_{a}\left(\vec{R}_{1}, \vec{R}_{2} \ldots, \vec{R}_{N}\right) P\left(\vec{R}_{1}, \vec{R}_{2} \ldots, \vec{R}_{N}\right)_{j} \prod_{1}^{N} d^{3} R_{j} \cdot I \cdot B \cdot 3
$$


The conditional probability $P\left(\vec{R}_{x} \mid \vec{R}_{1} \ldots \vec{B}_{12}-1 \cdot \vec{R}_{a}+1 \cdots \vec{R}_{N}\right)$ gives the probability of finding the remaining $N-1$ scatterers if the one at $\vec{R}_{\alpha}$ is held fixed, and is given by

$$
P\left(\vec{R}_{1}, \vec{R}_{2} \ldots \vec{R}_{N}\right)=P\left(\vec{R}_{a}\right) P\left(\vec{R}_{a} \mid \vec{R}_{1} \ldots \vec{R}_{a}-1 \cdot \vec{R}_{a}+1 \cdots \cdot \vec{R}_{N}\right) \text {, }
$$

where

$$
P\left(\vec{R}_{a}\right)=\operatorname{SP}\left(\vec{R}_{1}, \vec{R}_{2} \ldots, \vec{R}_{N}\right) j \prod_{a} d^{3} R_{j}
$$

Defining $\left.\boldsymbol{Q}_{\alpha}\right\rangle_{a}$ to be the conditional average of $Q_{\alpha}$

$$
\begin{aligned}
& \left\langle Q_{\alpha}\right\rangle_{\alpha}=s_{Q_{\alpha}}\left(\vec{R}_{1}, \vec{R}_{2} \ldots, \vec{R}_{N}\right) P\left(\vec{R}_{\alpha} \mid \vec{R}_{1} \ldots, \vec{R}_{\alpha}-1, \vec{R}_{\alpha}+1 \cdots \cdot \vec{R}_{N}\right) \\
& \text { j } a^{a^{3} R_{j}}
\end{aligned}
$$

Eq. I.B.3 can be written

$$
\langle T\rangle=\sum_{a} \rho\left\langle Q_{\alpha} ;{ }_{a} P\left(\vec{R}_{\alpha}\right) \mathbf{d}^{3} R_{\alpha}\right.
$$

Since each site in the system is equivalent, the sum over a contributes a factor of $\mathrm{N}$. The probability of finding a single ion is the same for $a 11 \vec{k}_{a}$ and $\mathrm{Eg}$. I.B.7 reduces to

$$
\langle T\rangle=\operatorname{MP}(\vec{R}) \rho\left\langle Q_{a}{ }_{a} d^{3} R_{a}=n \rho\left\langle Q_{a}\right\rangle_{a^{2}}{ }^{3} R_{a^{\prime}}\right.
$$

with $n$ the mean density. 
To calculate $\langle T\rangle$, one needs a means of evaluating the restricted average $\left\langle Q_{\alpha}\right\rangle_{\alpha}$. Recalling Eq. I.A 23, Eq. I.B.6 can be cast in the form

$$
\begin{aligned}
& \left.\left\langle Q_{\alpha}\right\rangle_{\alpha}=t_{\alpha}+t_{\alpha} G_{0}(2) \int t_{B} \sum_{\alpha} Q_{B}\right\} P\left(\vec{R}_{\alpha} \mid \vec{R}_{1} \ldots \vec{R}_{\alpha}-1 \cdots \vec{R}_{\alpha}+1^{\circ}\right. \\
& \left.\ldots, \vec{R}_{\mathrm{s}}\right)_{j} \mathbb{I}^{a^{3} R_{j}} \text {. }
\end{aligned}
$$

By making use of the conditional probabilities, one can reduce $\mathrm{Bq}$. I.B.9 to an integral of $\left(O_{G}\right\rangle_{B \alpha^{\prime}}$, the average of $Q_{\alpha}$ over all but two coordinates $\vec{R}_{\alpha}$ and $\vec{R}_{B}$. The conditional probability density with $\vec{R}_{\alpha}$ and $\vec{R}_{B}$ held fixes is related to that for site a held fixed by

$$
\begin{aligned}
& P\left(\vec{R}_{\alpha}\right) F\left(\vec{R}_{\alpha} \mid \vec{R}_{1} \ldots \ldots \cdot \vec{R}_{\alpha}-1 \cdot \vec{R}_{\alpha}+1 \cdots \cdot \vec{R}_{N}\right) \\
& =P\left(\vec{R}_{a}, \vec{R}_{B}\right) P\left(\vec{R}_{1}, \ldots, \vec{R}_{\alpha}-1, \vec{R}_{a}+1 \cdots, \vec{R}_{B}-1, \vec{R}_{B}+1 \cdots \cdots, \vec{R}_{H}\right) \cdot \\
& \text { I.B. } 10
\end{aligned}
$$

Combining Egs. I.B.9 and I.B.10 one obtains

$$
\left\langle Q_{a}\right\rangle_{a}=t_{a}+t_{a} G_{0}(2)(N-1) \int Q_{B} B a\left(P\left(\vec{R}_{a} \cdot \vec{R}_{B}\right) / P\left(\vec{R}_{a}\right) ! a^{3} R_{B}\right.
$$

The quantity $(\mathbb{N}-1) P\left(\vec{R}_{a}, \vec{R}_{B}\right) / P\left(\vec{R}_{a}\right)$ can be expressed as $n g\left(\vec{R}_{a}-\vec{R}_{B}\right)$ where $g\left(\vec{k}_{a}-\vec{k}_{B}\right)$ is the radial distribution function and gives the probability of finding an atomic ste at $\vec{Z}_{B}$ if one is known to be at $\vec{k}_{\alpha}$. Rq. I.B.11 becomes 


$$
\left\langle Q_{\alpha}\right\rangle_{\alpha}=t_{\alpha}+t_{\alpha} G_{0}(z) n ;\left(Q_{B}\right\rangle_{B a} g\left(\vec{R}_{\alpha}-\vec{R}_{B}\right) d^{3} R_{B} \cdot \quad \text { I.B.12 }
$$

Continuing along this line generates a similar relation between $\left\langle Q_{B}\right\rangle_{B a}$ and $\left\langle Q_{Y}\right\rangle_{Y B a}$ and so on. Approximations enter by introducing a procedure for decoupling averages from higher order averages.

The earliest, and simplest, decoupling scheme was proposed by Lax, 1951 (9) and is known as the quasicrystalline approximation (OCA). In Lax's description, $\left\langle Q_{\beta}\right\rangle_{B Q}$ is replaced by $\left\langle Q_{B}\right\rangle_{B}$ and Eq. I.B.12 reduces to

$$
\left\langle Q_{\alpha}\right\rangle_{\alpha}=t_{\alpha}+t_{\alpha} G_{0}(z) n \int\left\langle Q_{\beta}\right\rangle_{B} g\left(\vec{R}_{\alpha}-\vec{R}_{B}\right) d^{3} R_{B} \text {, I.B.13 }
$$

which corresponds to truncating the hierarchy of equations of the type Eq. I.B.12 at first order. By iterating Eq. I.B.13 the series

$$
\begin{aligned}
\left\langle Q_{\alpha}\right\rangle_{\alpha} & =t_{\alpha}+t_{\alpha} G_{0}(z) n \int g\left(\vec{R}_{\alpha}-\vec{R}_{B}\right) t_{B} d^{3} R_{B} \\
& +t_{\alpha} G_{0}(z) n \int g\left(\vec{R}_{\alpha}-\vec{R}_{\beta}\right) t_{B} G_{0}(z) n \int g\left(\vec{R}_{B}-\vec{R}_{\gamma}\right) t_{\gamma} d^{3} R_{\gamma} d^{3} R_{B}+\ldots
\end{aligned}
$$

results. Por certain potentials, this series can be sumed in closed form in a momentum representation. Eq. I.B.14 can be inserted in $\mathrm{Eg}$. I.B.8 to obtain $\langle\mathrm{T}\rangle$ which in turn determines $\langle G(z)$; according to Eq. I.B.1. 
An alternative procedure for decoupling Eq. I.B.12 was suggested by Schwartz and Enrenreich, 1971 (10). They introduce the self-energy $\Sigma(2)$ defined such that

$$
\langle G(z)\rangle=\left(z-H_{0}-\Sigma(z)\right)^{-1}
$$

and the local self-energy for the $a^{\text {th }}$ site $\sigma_{a}$

$$
\sigma_{a}=\omega_{a} ; G_{0} G^{-1}
$$

$\Sigma(2)$ is obtained from $\sigma_{a}$ according to

$$
\Sigma(z)=n \int \sigma_{\alpha} d^{3} R_{\alpha} .
$$

Their decoupling approximation can be shown to be

$$
\left\langle Q_{B}\right\rangle_{B Q} G_{0}(z)=\left\langle Q_{B}\right\rangle_{B} G_{0}(z)\langle G(z)\rangle^{-1}\langle G(z)\rangle_{\alpha^{\prime}}
$$

where

$$
\langle G(2)\rangle_{\alpha}=\int G\left(\vec{R}_{1}, \vec{R}_{2}, \ldots, \vec{R}_{N} ; 2\right) P\left(\vec{R}_{\alpha} \mid \vec{R}_{1}, \vec{R}_{2}, \ldots, \vec{R}_{N}\right) ; \prod_{a} d^{3} R_{j}
$$

and obeys the relation

$$
\langle G(z)\rangle_{\alpha}=\langle G(z)\rangle+\langle G(z)\rangle \bar{t}_{a}\langle G(z)\rangle .
$$

The operator $\bar{t}_{a}$ is a rather complicated operator, however upon defining the quantity 


$$
\left.\tilde{\sigma}_{\alpha}=-n \int ! g\left(\vec{R}_{\alpha}-\vec{R}_{B}\right)-1\right] \sigma_{B} d^{3} R_{B}, \quad \text { I.B.21 }
$$

a pair of self-consistent equations are developed for $\bar{t}_{\alpha}$ and $J_{\alpha}$ in terms of $\langle G(z))$ and $v_{\alpha}$, the potential on site $\alpha$.

$$
\sigma_{\alpha}=v_{\alpha}+v_{\alpha}\left\langle G(z) \bar{t}_{\alpha}, \quad\right. \text { I.B.2z }
$$

and

$$
\bar{t}_{\alpha}=\left(v_{\alpha}-\tilde{\sigma}_{\alpha}\right)+\left(v_{\alpha}-\tilde{\sigma}_{\alpha}\right)\left(G(2) \bar{t}_{\alpha} .\right.
$$

Eqs. I.B.22 and I.B.23 are the principal equations of what Schwartz and Ehrenreich call the self-consistent approximation (SCA). This method is self-consistent in the sense that $\sigma_{a}$ and $\bar{t}_{\alpha}$ are determined in terms of the configuration averaged propagator $\left\langle G(z)\right.$; which itself depends upon $\sigma_{a}$ through $\Sigma(z)$. Schwartz and Ehrenreich have shown that the decoupling procedure used to obtain the SCA leads to the CPA when applied to a substitutional binary alloy.

Attempts have been made to develop a direct extension of the CPA to the liquid metal problem. Faulkner, 1970 (11) has constructed the CPA for a liquid metal in the absence of short-range order and found it to be equivalent to results obtained by $k 1$ auder (12) using a perturbation theoretic treatment. Gyorffy, 1970 (13) has generalized the CPA for liquids to include short-range order, developing a 
self-consistent treatment which predated the SchwartzEhrenreich scheme mentioned above. Gyorffy's treatment was later refined and extended by Korringa and Mills, 1972 (14). The treatment of Korringa and Mills is described below. Again, the Hamiltonian of interest is $H=B_{0}+\sum_{i} V_{i}$ with $G(z)=(2-H)^{-1}$ and

$$
\langle G(2)\rangle=\left[2-H_{0}-w(2)\right]^{-1}
$$

where $W(z)$ is the coherent potential to be determined. By defining $H_{1}$ and $T$ such that

$$
H_{1}=\sum_{i} V_{i}-W(z),
$$

and

$$
T\langle G(z)\rangle=H_{1} G(z) \text {. }
$$

one can write

$$
G(z)=\langle G(z)\rangle+\langle G(z)\rangle T\langle G(z)\rangle .
$$

Averaging both sides of Eq. I.B.27 requires

$$
\langle\mathbf{T}\rangle=0 \text {. }
$$

Korringa and Mills introduce the operators $\hat{T}$ and $-q$ as the local and nonlocal contributions, respectively, to $T$ 


$$
\mathbf{T}=\hat{\mathbf{T}}-\mathbf{q} .
$$

A bit of algebra reveals that

$$
q=w(z)+w(z)\langle G(z)\rangle T,
$$

from which it follows that

$$
\langle q\rangle=\langle\hat{T}\rangle=w(z) .
$$

Writing $\hat{\mathbf{T}}=\sum_{\hat{i}}^{\mathcal{O}_{i}}$ with the defining relation

$$
Q_{i}=v_{i}+v_{i}\langle G(z)\rangle_{\text {, }}
$$

one can demonstrate that

$$
Q_{i}=t_{i}+t_{i}\left\langle G(z) ; Q_{j} Q_{j}-q\right) .
$$

Multiplying Eq. I.B.33 by $s\left(\vec{R}-\vec{R}_{i}\right)$, averaging, suming over $i$, and defining the mean density $\rho(\vec{R})$ and the mean local operator $Q(\vec{R})$.

$$
\rho(\vec{R}) Q(\vec{R})=\sum_{i}\left(\vec{R}-\vec{R}_{i}\right) Q_{i} ;
$$

ore obtains

$$
\begin{aligned}
\rho(\vec{R}) Q(\vec{R})= & \rho(\vec{R}) t(\vec{R})+t(\vec{R})\left\langle G(z) j \int d^{3} R^{\prime} \sum_{i} j i\right. \\
& \left\langle j\left(\vec{R}-\vec{R}_{i}\right) \delta\left(\vec{R} \cdot-\vec{R}_{j}\right) Q_{j} ;\right. \\
& \left.-t(\vec{R})\langle G(z)\rangle \sum_{i} \delta\left(\vec{R}-\vec{R}_{i}\right) q\right) .
\end{aligned}
$$


where $g\left(\vec{R}-\vec{R}^{\prime}\right)=g\left(\vec{R}_{,} \vec{k}^{\prime}\right)$ is the radial distribution function defined earlier in this section. Substitution of Eqs. I.B.37 and I.B.38 into Eq. I.B.35 and defining $\bar{g}\left(\vec{R}-\vec{R}^{\cdot}\right)=$ $g\left(\vec{R}-\vec{R}^{\prime}\right)$ - $\rho\left(\vec{R}^{\prime}\right)$ (following Korringa and Mills) one finds

$$
Q(\vec{R})=t(\vec{R})+t(\vec{R}) G(z) \iint d^{3} \vec{R} \cdot \vec{g}\left(\vec{R}-\vec{R}^{0}\right) Q(\vec{R} \cdot) . \quad I . B .39
$$

Along with the definition of $t(\vec{R})$

$$
\rho(\vec{R}) t(\vec{R})=\sum_{i}\left\langle\delta\left(\vec{R}-\vec{R}_{i}\right) t_{i}\right.
$$

kiqs. I.B.24, I.B.36, and I.B.39 constitute a self-consistent scheme for obtaining $(G(z)$.

of the above thiee schemes for computing the electronic properties of liquid metals, only the non-self-consistent OCA is easily applied to even simple model systems. Other approximations have beer. suggested to treat the liquid metal prob!em, but they are more complicated than those mentioned above. Notable among these aie the effective-medium approximation of Roth, 1974 (15) and the approximation of Ishida and Yonezawa, 1973 (16).

\section{c. Numerical Investigations}

Along with attempts to develop suitable approximate descriptions of the properties of excitations in cisordered systems, considerable effort has gone into numerical 
calculaticns on nodel systers. These model calculations are useful for evaluating suggested approximations and for pointing out features related to the effects of disorder. A review of the numerical work on vibrational systens has been given by Dean (17).

In a recent study of the electronic states in a one-dimensional liquid netal, Peterson, Schwartz, and Butler (PSB) (18) reported a comparison of the exact integrated density of states $n(E)$ to calculations of $n(E)$ from three approximate theories. The approximations considered in that work were the self-consistent theories of Schwartz and Ehrenreich (10) and Gyorffy (i3) (as extended by Korringa and Mills (14), and the non-self-consistent quasicrystalline approximation of Lax (9). The basic elements of these theories were discussed in the preceding section. Somewhat surprisingly, $n(E)$ obtained from the QCA and $n(E)$ obtained from the self-consistent theories all gave about the same level of agreement with the exact results. Each theoretical description gives wery good agreenent with the exact results in the weak-scattering regime, but none of them provides an adequate description in the - crong-scattering regime.

Numelical investigations of model systems have generally been directed toward calculating either the density of states or the integrated density of states. There have been relatively few calculations of momentum dependent quantities such as the spectral density $\lambda(\vec{k}, E)$ defir:t as 


$$
A(\vec{k}, E)=-\frac{1}{\pi} I n G(\vec{k}, E) . \quad I . C .1
$$

where $G(\vec{k}, E)$ is the Fourier transform of the one-particle Green's function. Recently a nusical calculation of the spectral density function for eltctronic excitations in a binary alloy has been presented by Alben, Blue, Krakauer. and Schwartz (19). Comparing their results with the CPA results for the same systen shored the CPA in excellent agreement. The same technique has been applied by Alben and Thorpe (20) to a calculation of the spin-wave spectrum in a randow antiferromagnet.

The earliest calculation of $A(\vec{k}, E)$ for an exactly solvable model was reported by Halperin (21) in 1965. Halprin developed a formalism for calculating the spectral density for a particle noving in a one-dimensional whiteGaussian-noise" potential. The white-Gaussian-noise potential is so termed because the Pourier components of the potentiai have a Gassian distrisution and no correlations exist between fourier components at different freavencies. No short-range order is included in such a mode:.

The chapters which follow describe al adaptation of Halperin's formalism to Honte Carlo calculations of the spectral density $A(k, E)$ for elementary excitations in amcrphous systens with short-range order. Several Haniltonians have been studied and the results of the calculation have 
been compared to the spectral density obtained in the quasicrystalline approximation. The qualitative features of recent neutron scattering measurements are interpreted based on these calculations. 


\section{CAMPTER II}

TIE SPECTRAL DEASITY FOR AI EIECTMOA IN A OAE-DIRLASIOWN LIQUID RETAL

This chapter describes a Monte Carlo calculation of the spectral density function $A(k, E)$ for an electron woving in a one-dimensional model of a liquid metal. This study is complementary to the calculation of the exact integrated density of states by Peterson, Schwartz, and Butler (PSB) (18) mentioned in the previous chapter. The exact $A(k, E)$ will be compared to that calculated within the quasicrystalline approximation (OCA) of Lax (9). Part A descri'ses the onedimensional model, Part B gives the prosedure for calculating $A(k, E)$. Part $C$ discusses the convergence of the spectral density, and Part D outlines the properties to be expected of the spectral density for a disordered system and compares the QCA and exact numerical results.

\section{A. The Model System}

The statistical model chosen for this study is the so-called hard rod model of the classical one-dimensional fluid (22). This model has been used by PSB (18). The model can be pictured as the one-dimensional equivalent of the hard-sphere model often used in three-dimensional treatments of liguids and gases. N identical line segments 
of length a are distributed on a line of length N! subject to the condition that they do not overlap. The atomic positions are taken to be at the centers of these segments. The mininu separation between nearest-neighbors is the length a and the average separation is the leagth $l$. The hard-rod distribution is illustrated in Figure 1. The parameter $a=a / l$ is a measure of the amount of disorder in che systen with $a=1$ indicating perfect order, $a=0$ indicating complete disorder (Poisson distribution), and suitable choices of a indicating internediate levels of disorder. Quantitatively, the probability of finding a nearest-neighbor at a distance d from a given atom is

$$
p(d)=\frac{\theta(d-a)}{l-a} \exp \left\{-\frac{d-a}{l-a}\right\} \quad \text { II.A.I }
$$

where $\theta(x)$ is zero for $x<a$ and one for $x>a$.

The interaction between an electron and the system of $N$ atoms is described by the potential

$$
v(x)=-\Delta \sum_{j=1}^{N} \delta\left(x-x_{j}\right), \quad I I . A .2
$$

where $x_{j}$ is the position of the $j^{\text {th }}$ atom and $\Delta$ is the strength of the delta function poientials. The quantities $a_{j}=x_{i}-x_{j-1}$ are random quantities obtained from a random number generating subroutine consistent with Rq. II.A.1. 


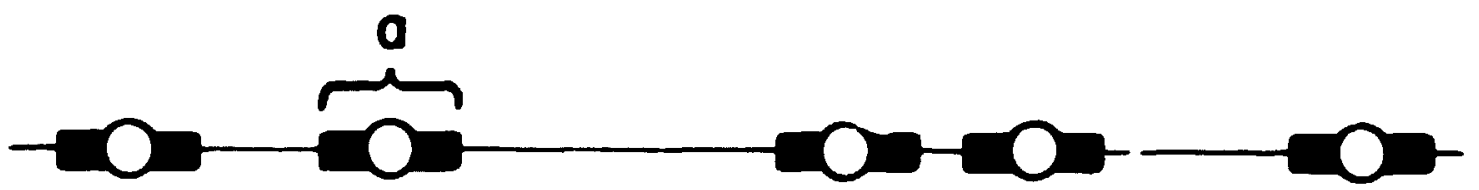

Figure 1. Schenatic representation of the hard-rod Iiquid. 
Let $R_{j}$ be a random variable distributed uniformly on the interval $(0,1)$. The random variable $d_{j}$ with probability density $p\left(d_{j}\right)$ is obtained from the equation

$$
R_{j}=\int_{\mathbf{j}}^{d_{j}} p(y) d y .
$$

Por the hard-rod distribu ion, this becomes

$$
C_{j}=a+i(a-1) \ln \left(1-R_{j}\right)
$$

and can be used to construct the one-dinensional model described above.

\section{B. Calculation of the Spectral Density}

The spectral density $A(k, E)$ was defined in Eq. I.C.I. It is related to the density of states by

$$
\rho(E)=\frac{1}{2 \pi} \rho A(k, E) d k \text {. }
$$

in one dimension. $A(k, E)$ can be written in terms of the one-electron wavefunctions $j(x)$

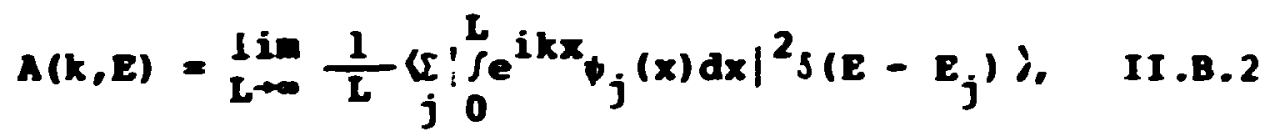

where the wavefunctions are solutions of the Schrodinger equation 


$$
1-\frac{d^{2}}{d x^{2}}+v(x) j \psi_{j}(x)=E_{j} \psi_{j}(x) \text {. }
$$

with V(x) given in Eq. II.A.2. Halperin (21) has reduced the calculation of $\mathrm{A}(\mathrm{K}, \mathrm{E})$ to a simpler form than Eq. II.B.2. This procedure is described below.

Let us define for each potentia: consiguration in the statistical ense-ble a function $\diamond(x ; E)$ to be the unique solution of Eq. II.B.3 for energy $E$ subject to the boundary conditions

$$
\left.\frac{\partial \hat{\theta}(x ; E)}{\partial x}\right|_{x=0}=2_{\theta} \phi(0 ; E) .
$$

and

$$
\left.\frac{\partial \varphi(x ; E)}{\partial x}\right|_{x=L}=z_{L} \phi(L ; E)
$$

where $z_{0}$ and $z_{I}$ are arbitrary constants. Halperin defines the functions

$$
z(x ; E)=\frac{\partial \phi(x ; E)}{\partial x} / \phi(x ; E) .
$$

and

$$
U_{1}(x ; E)=\int_{0}^{x} e^{i k x^{\prime}} \downarrow\left(x^{\prime} ; E\right) d x^{\prime} \mid / e^{i k x} \phi(x ; E)
$$

II .B.7 
in terms of which $A(k, E)$ is to be written, and the two auxiliaxy functions

$$
U_{2}(x ; E)=\left\{\int_{0}^{x} \phi^{2}\left(x^{\circ} ; E\right) d x^{\circ}\right\} / \phi^{2}(x ; E) . \quad \text { II.B.8 }
$$

and

$$
v_{3}(x ; E)=\frac{\partial z(x ; E)}{\partial x}
$$

The last two functions are solutions of the equations

$$
\frac{\partial U_{2}(x ; E)}{\partial x}=1-22(x ; E) U_{2}(x ; E) . \quad \text { II.B. } 10
$$

and

$$
\frac{\partial U_{3}(x ; E)}{\partial x}=-1-2 z(x ; E) U_{3}(x ; E)
$$

From these it can be shown that

$$
U_{3}(x ; E)=-U_{2}(x ; E)
$$

fur all $x$ and since $U_{2}(x ; E)$ is positive, $U_{3}(x ; E)$ must be a negative quantity. Using tr.s scaling properties of the delta-functions, it is easily demonstrated that

$$
\sum_{i} \delta\left(E-E_{i}\right)=\left|\frac{\partial z\left(L_{\&} E\right)}{\partial E}\right| \delta\left\{z_{L}-z(L ; E)\right\} .
$$


Upon waking the appropriate substitutions in Eq. II.B.2 one obtains

$$
\begin{aligned}
& A^{L}(K, E)=\frac{1}{L} d \mid U_{1}(E ; E)^{2} / U_{2}(L ; E) \underset{i}{\mid \sum \delta(E-E}-E_{i} ; \quad I I . B .14 \\
& A^{L}(K, E)=\frac{1}{L}\left\langle\{ | U _ { 1 } ( L ; E ) | ^ { 2 } / U _ { 2 } ( L ; E ) \} \left\{ U_{3}(L ; E) \mid \delta\left\{z_{L}-2(L ; E)\right\} j .\right.\right. \\
& \mathbf{A}^{L}(k, E)=\frac{1}{L}\left\langle\left|U_{1}(L ; E)\right|^{2} \delta\left\{z_{L}-2(L ; E)\right\}:\right.
\end{aligned}
$$

If the function $(x ; E)$ can be constructed, Eq. II.B.16 provides a means of calculating $A(k, E)$.

Suppressing womentarily the arguments of $v_{1}$ and $z$, the average in Eq. II.B.16 can be written as

$$
A^{L}(k, E)=\frac{1}{L} \int S\left|u_{1}\right|^{2} \delta\left(z_{L}-z\right) P\left(u_{1}, z\right) d U_{1} d z, \quad I I .0 .17
$$

where $P\left(U_{1}, z\right)$ is the joint probability density for the random variables 2 and $U_{1}$. Performing the z-integral gives

$$
A^{L}(k, E)=\frac{1}{L} \int\left|v_{1}\right|^{2} P\left(v_{1}, z_{L}\right) d u_{1}
$$

As I becomes large, $\Lambda^{L}(k, E)+A(k, E)$ and must be independent of the arbitrary constant $z_{L}$. The probability density $P\left(U_{1}, z_{L}\right)$ must approach $P\left(U_{1}\right)$, a quantity independent of $z_{L}$ as $\mathrm{l} \rightarrow$. The spectral density, then, is given by 


$$
A(K, E)=\lim _{L \rightarrow \infty} \frac{1}{L}\left\langle\left|U_{1}(L ; E)\right|^{2}\right.
$$

The determination of $A(K, E)$ in Eq. II.B.19 can be further simplified by taking advantage of the properties of the delta function potentials indicated in Eq. II.A.2. Let $A(x)$ be defined such that

$$
A(x)=\left\{i k \phi(x ; E) e^{i k x}-e^{i k x} \phi^{\prime}(x ; E)\right\} \quad \text { II .B. } 20
$$

with $\Phi(x ; E)$ the solution of the Schrodinger equation as given in Eq. II.B.3. Differentiating $A(x)$ and substituting for the second derivative $\phi^{\prime \prime}(x ; E)$ from the Schrodinger equation yields

$$
\frac{d}{d x} A(x)=e^{i k x} \phi(x ; E)\left(E-k^{2}\right)-e^{i k x} v(x) \phi(x ; E)
$$

II.B. 21

Integrating both sides and rearranging terms provides the form

$$
\int_{0}^{L} e^{i k x} \phi(x ; E) d x=\frac{1}{\left(E-k^{2}\right)}\left[\int_{0}^{l} e^{i k x} v(x) \phi(x ; E) d x+\left.A(x)\right|_{0} ^{L}\right]
$$

Dividing both sides by $e^{j k L} \phi(\tau ; E)$ and recalling the definition of $U_{1}(x ; E)$ one obtains 
$u_{1}(L ; E)=\frac{1}{\left(E-k^{2}\right)}\left[\frac{\int_{0}^{L} e^{i k x} v(x) \phi(x ; E) d x}{e^{i k L_{\phi(L ; E)}}}+\left.\frac{A(x)}{e^{i k L_{\phi(L ; E)}}}\right|_{0} ^{L}\right]$

II.B. 23

Inserting the definition of $V(x)$ for this model in the above equation and performing the iricegration leads to the result

$$
\begin{aligned}
u_{1}(L ; E)= & \frac{1}{\left(E-k^{2}\right)}\left[\frac{-\Delta \sum_{j}^{N} \exp ^{\left.N i k x_{j}\right\} \phi\left(x_{j} ; E\right)}}{\exp (i k L\} \phi(L ; E)}\right. \\
& +\frac{A(x)}{\left.\left.e^{i k L_{\phi(L ; E)}}\right|_{0} ^{L}\right]}
\end{aligned}
$$

The second term in the above equation can be written explicitly as

$$
\begin{aligned}
& \left.\frac{A(x)}{\exp (i k L) \phi(L ; E)}\right|_{0} ^{L} \\
& =i k-z(L ; E)-e^{-i k L}\left\{i k-\frac{(0 ; E)}{\phi(L ; E)}-\frac{\phi^{\prime}(0 ; E)}{\phi(L ; E)}\right\} \cdot I I . B .25
\end{aligned}
$$

The amplitude of the wavefunction $\phi(x ; E)$ grows exponentially with increasing $x$ and the term in curly brackets in Eq. II.B.25 becomes negligible in a numerical calculation $(23,24)$. The final expression for $U_{1}(L ; E)$ is 


$$
\begin{aligned}
u_{1}(L ; E)= & \frac{1}{\left(E-k^{2}\right)}\left[\frac{-\Delta \sum^{\sum} \operatorname{lxp}^{N}\left(i k x_{j}\right) \phi\left(x_{j} ; E\right)}{\exp (i k L) \phi(1, ; E)}\right. \\
& +i k-z(L ; E)]
\end{aligned}
$$

Substituting this in Eq. II.B.19 provides an expression for evaluating the spectral density in terms of the values of the wavefunctions at the sites $x_{j}$.

The wavefunctions $\phi\left(x_{j} ; E\right)$ can be constructed wy a transfer matrix method. The interval $x=0$ to $x=L$ is divided into $N$ cells. The $i^{\text {th }}$ cell is that region of the $x$-axis bounded by the delta functions at $x_{i-1}$ on the left and $x_{i}$ on the right. In the $i^{\text {th }}$ cell, the wavefunctions have the form

$$
\Phi_{i}(x ; E)=A_{i} e^{i Y x}+B_{i} e^{-i \gamma x} .
$$

(real wavefunctions could also be used) where $E=\gamma^{2}$. The continuity conditions on $\phi_{i}(x ; E)$ are $(25)$

$$
\phi_{i}+1\left(x_{i} ; E\right)=\phi_{i}\left(x_{i} ; E\right)
$$

and

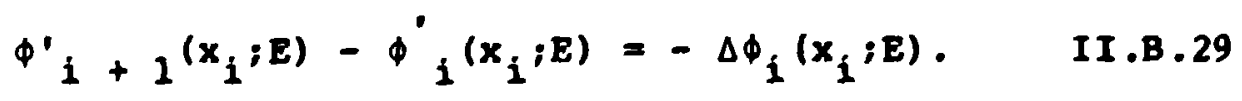


A transfer matrix $\boldsymbol{H}$ can be defined which carries the values of ${ }^{\prime}{ }_{i}\left(x_{i-1} ; E\right)$ and $\phi_{i}\left(x_{i-1} ; E\right)$ into the values ${ }^{\prime}{ }_{i}\left(x_{i} ; E\right)$ and $\phi_{i}\left(x_{i} ; E\right)$.

$$
\left[\begin{array}{l}
\phi_{i}^{\prime}\left(x_{i} ; E\right) \\
\phi_{i}\left(x_{i} ; E\right)
\end{array}\right]=\left[\begin{array}{ll}
H_{11} & M_{12} \\
H_{21} & M_{22}
\end{array}\right]\left[\begin{array}{l}
{ }_{i}^{\prime}\left(x_{i-1} ; E\right) \\
\phi_{i-1}\left(x_{i-E)}\right.
\end{array}\right]
$$

II .B. 30

Application of the matching conditions defined above to the wavefunction in Eq. II.B.27 yields the matrix

$$
M=\left[\begin{array}{lc}
\cos \left(\gamma d_{i}\right) & -\gamma \sin \left(\gamma d_{i}\right) \\
\frac{1}{\gamma} \sin \left(\gamma d_{i}\right) & \cos \left(\gamma d_{i}\right)
\end{array}\right] .
$$

The two equations contained in the matrix equation can be written explicity as

$$
\phi^{\prime}{ }_{i}\left(x_{i} ; E\right)=\cos \left(\gamma d_{i}\right) \phi^{\prime}{ }_{i}\left(x_{i-1} ; E\right)-\gamma \sin \left(\gamma d_{i}\right) \phi_{i}\left(x_{i-1} ; E\right)
$$

and

$$
\phi_{i}\left(x_{i} ; E\right)=\frac{1}{\gamma} \sin \left(Y d_{i}\right) \phi^{\prime}{ }_{i}\left(x_{i-1} ; E\right)+\cos \left(\gamma d_{i}\right) \phi_{i}\left(x_{i-1} ; E\right) .
$$

II .B. 33

Dividing $\mathrm{Eg}$. II.B.33 by Eq. II.B.32 and applying the matching conditions on the wavefunction and its derivative yields 


$$
z_{i+1}\left(x_{i} ; E\right)=\frac{z_{i}\left(x_{i-1} ; E\right)-r \tan \left(Y d_{i}\right)}{\frac{1}{y} \tan \left(r d_{i}\right) z_{i}\left(x_{i-1} ; E\right)+1}-\Delta \quad \text { II.B.34 }
$$

an expression which can be used to generate a sequence of values of the logarithaic derivatives $z_{i+1}\left(x_{i} ; E\right)=$ ${ }^{\prime}{ }_{i+1}\left(x_{i} ; E\right) / \Phi_{i+1}\left(x_{i} ; E\right)$ in terms of the energy, the strength of the potential, and the random variable $d_{i}$. Eq. II.B.33 can be recast in the form

$$
\uparrow_{i}\left(x_{i} ; E\right)=\$_{i-1}\left(x_{i-1} ; E\right)\left[\frac{1}{\gamma} \sin \left(\gamma d_{i}\right) z_{i}\left(x_{i-1}\right)+\cos \left(\gamma d_{i}\right)\right] \text {. }
$$

and can be used to provide the wavefunctions needed to evaluate $A(k, E)$ numerically.

\section{Convergence of $A(k, E)$}

Eq. II.B.19 shows that the configuration averaged spectral density $A(K, E)$ is the mean value of the random variable $f_{L}(K, E)=\frac{1}{L}\left|U_{1}(L ; E)\right|^{2}$. It remains tc be shown how one calculates that average value. The configuration averaged integrated density of states $n(E)$ is related to the average nimber of nodes per unit length of $(x ; E)(26)$. The number of nodes per unit length of $\phi(x ; E)$ for a onedimensional system converges on its average value with zero variance as the length of the system increases. The number 
of nodes per unit length is, then, an ergodic quantity (in the statistical sense) and $n(E)$ can be determined by a node-counting technique on a single long chain of atoms. The remainder of this section will be devoted to an analysis of the convergence properties of the random variable $f_{L}(k, E)$. It will be demonstrated that $f_{L}(K, E)$ is not ergodic (27) (in the statistical sense) and that the mean of the distribution of $f_{L}(K, E)$ cannot be calculated by calculating $f_{L}(x, E)$ for a single long chain of atoms.

The ratio $g(x ; E)=\Phi(x ; E) / \phi(L ; E)$ is a random process for $x \geq 0$ and is assumed for simplicity to be real. Let us define the random function of $k$ and $E$

$$
f_{L}(k, E)=\frac{1}{L}\left|\int_{0}^{L} e^{i k x} g(x ; E) d x\right|^{2}
$$

for a system of length L. The spectral density is given by

$$
A(k, E)=\lim _{L \rightarrow \infty}\left\langle f_{L}(k, E) i .\right.
$$

Eq. II.C. 2 can be put in the form

$$
A(K, E)=\lim _{L \rightarrow \infty} \frac{1}{L} \int_{0}^{L} \int_{0} e^{i k(x-y)} g(x ; E) g(y ; E) \lambda d x d y .
$$

II.C. 3 
The quantity in averaging bracket: is the correlation function of the randon process $g(x ; E)$. It is assumed that this correlation function depends on the difference $x-y$.

$$
R(x-y)=\langle g(x ; E) g(y ; E):
$$

and the spectral density becomes

$$
A(k, E)=\lim _{L \rightarrow \infty} \frac{1}{L} \int_{0}^{L} \int_{0}^{L} e^{i k(x-y)} R(x-y) d x d y . \quad I I . C .5
$$

This demonstrates that the configuration averaged spectral density $A(\mathrm{~K}, \mathrm{E})$ is given by the Fourier transform of the correlation function $R(x-y)$. Albegraic manipulation of Eq. II.C.5 (see Appendix A) noting that $R(x-y)=R(y-x)$ yields

$$
A(k, E)=\lim _{1 \rightarrow \infty} 2_{0}^{L}\left(1-\frac{11}{L}\right) \cos (k u) R(u) d u, \quad \text { II.C.6 }
$$

which is readily reduced to give

$$
A(k, E)=2 \int_{0}^{\infty} \cos (k u) R(u) d u \quad \text { II.C.7 }
$$

Eq. II.C.7 is a convenient expression for the spectral density for the discussion which follows.

The random variable $f_{L}(k, E)$ has the average value $A(k, E)$ is the limit $\mathrm{L} \rightarrow \infty$. To be a consistent estimator of the 
spectral density, $f_{L}(k, E)$ must, for each $k$ and $E$, approach $A(k, E)$ with zero variance as $L$ increases to infinity. That this is not the case in general can be demonstrated by a simple exanple. Let $g(x ; E)$ be a normal process with wean zero. The objective is to calculate the variance $\sigma^{2}$ of $f_{L}(k, E)$ as $L+\infty$. The variance is defined by the relation

$$
\sigma^{2}=\left\langle\left(f_{L}(k, E)-\left\langle f_{L}(k, E)\right\rangle\right\}^{2} ;\right.
$$

or

$$
\sigma^{2}=\left\langle\left\{f_{L}(k, E)\right\}^{2} ;-\left\langle f_{L}(k, E)\right\rangle^{2}\right.
$$

The first of the two quantities on the right-hand side if Eq. II.C.9 is given by

$$
\left\langle\left(f_{L}(K, E)\right\rangle^{2}\right\rangle=
$$

$\frac{1}{L^{2}} \int_{0000}^{L L L L} e^{i k(w-x+y-z)} g(w ; F) g(x ; E) g(y ; E) g(z ; E) j$ dwdxdydz.

A tedious but straightforward calculation utilizing the pr'sperties of normally distributed random variables provides the result (see Appendix $u$ )

$$
\lim _{L \rightarrow \infty}\left\langle\left\{f_{L}(k, E)\right\}^{2}\right\rangle=2 \lim _{L \rightarrow \infty}\left\langle f_{L}(k, E)\right\rangle^{2},
$$


from which it follows that

$$
\sigma^{2}=\lim _{L \rightarrow \infty}\left\langlef _ { L } \left( k, E L^{2}=\Lambda^{2}(k, E)\right.\right.
$$

This exanple shows that $f_{L}(K, E)$ in the limit $L+\infty$ is not a self-averaging quantity, but is a randon variable with unknown distribution and average value $A(k, E)$. For the simple statistics chosen for this example, the proof can be carried one step further to show that $f_{L}(K, E)$ for large $L$ is exponentially distributed with mean value $A(k, E)(28)$. In general, the distribution function cannot be found and $A(k, E)$ can only be obtained by calculating $f_{L}(k, E)$ for several long chains. This amounts to generating several values of $f_{L}(k, E)$ consistent with the unknown distribution function and averaging the results to obtain $A(K, E)$.

The above discussion should not be interpreted to mean that for a given system of length $L(L+\infty)$ the spectral density has no meaning. $f_{L}(K, E)$ is not the spectral density for a particular system, it is a random variable whose mean value is the spectral density. In fact, there does exist a means of obtaining $A(k, E)$ by constructing only a single long chain of atoms (27). Consider a single system of length $I$ with $I$ large. This system can be broken up into n segments of length $L_{0}$. Let us define the quantities 


$$
\hat{f}_{L}(k, E)=\left.\frac{1}{L} \int_{(j-1) L_{0}}^{j L_{0}} e^{i k x_{g}(x ; E) d x}\right|^{2}
$$

and

$$
\hat{A}(K, E)=\frac{1}{n} j \stackrel{K}{E}_{1} \hat{\mathbf{E}}_{j}(K, E)
$$

Performing an analysis of $\hat{A}(k, E)$ similar to that described above gives the mean value $M\{\ldots\}$ and variance $v\{\ldots\}$ to be

$$
\lim _{\mathbf{L}_{0}+\infty} \operatorname{M}\{\hat{A}(k, E) ;=A(k, E)
$$

and

$$
\lim _{\substack{n \rightarrow \infty \\ \mathbf{L}_{0} \rightarrow \infty}} V(\hat{A}(k, E)\}=0
$$

The quantity $\hat{A}(K, E)$ is, then, a consistent estimator of $A(k, E)$ for large $n$ and $L_{0}$ and requires that one construct only a single chain of atoms. Ihis method of finding $A(K, E)$ has practical disadvantages, however. As mentioned in Part $B$ of this chapter, the wavefunction $\phi(x ; E)$ grows exponentially with $x$ and the 1 imit of large $n$ with large $I_{0}$ could not be achieved. Therefore, in this work, $A(K, E)$ is determined by calculating $f_{L}(K, E)$ for several chains and averaging the results. 


\section{Comparison of the QCA and Exact Results}

The general features to be expected of $A(k, E)$ for a disordered system can be understood by considering the properties of the correlation. function $R(u)$. In general, the spectral density is relaced to the correlation function by (see Eq. II.B.7)

$$
A(k, z)=\int_{-\infty}^{\infty} e^{-i k u} R(u) d u,
$$

where $R(u)$ depends upon $E$. Borland (23) has shown that the eigenfunctions for a one-dimensional disordered system are exponentiaily localized. Let us assume that the wavefunction is exponentially localized at the origin with an oscillatory component described by a single cosine factor. The correlation function then has the form

$$
R(u)=\left\langle g^{2}(0 ; E)\right\rangle e^{-\rho u} \cos (B u) \text {, }
$$

or

$$
R(u)=\sigma_{g}^{2} e^{-\rho u} \cos (B u)
$$

The spectral density is obtained by performing the integration in Eq. II.D.1.

$$
A(k, E)=\sigma_{g}^{2}\left[\frac{\rho}{(k-B)^{2}+\rho^{2}}+\frac{\rho}{(k+B)^{2}+\rho^{2}}\right] \cdot \text { II .D.4 }
$$


In a periectly ordered system, the electronic states are extended and $\theta=0$. Taking the Iinit $p+0$ in Eq. II.D.4 and using the identity

$$
\delta\left(x-x_{0}\right)=\frac{1}{n} \lim _{n \rightarrow 0} \frac{n}{\left(x-x_{0}\right)^{2}+n^{2}} . \quad \text { I.D.5 }
$$

gives the ordered-s, iten spectral density

$$
\lambda(k, E)=\pi \sigma_{g}^{2}[\delta(k-B)+5(k+B)] . \quad \text { II.D.6 }
$$

as a pair of delta functions. For a aisordered system, the delta functions become broadened into Lorentzian lineshapes. If $\rho$ is very large, corresponding to a highly localized state. the spectral density will be very broad and flat and may wash out entirely. The parameters $\sigma_{g^{\prime}} \rho$, and $B$ depend upon the energy and the potential, and would be difficult to calculate for even a simple model. Nevertheless, the example cited above leads one to expect broadening of the peaks in $A(k, E)$ to be a general feature for disordered systems.

The formal details of the quasicrystalline approximation (QCA) were given in Chapter I. The QCA has been applied to the model of Chapter II, Part A, by PSB (18). In what follows, the basic equations of the QCA are presented and the reader is referred to reference 18 for further details. Within the QCA, the spectral density is obtained according to Eq. I.C.1 from the Green's function 


$$
G(k, E)=\left[E-k^{2}-[(k, E)]^{-1}\right.
$$

where $[(k, E)$ is the self-energy. The numerical ingredients for determining $\Sigma(k, E)$ are $g^{-}(k)$, the fourier transform of the two-particle distribution function lobtained from the hard-rod distribution function, see PSB)

$$
g^{-}(k)=\frac{e^{i k a}}{1-e^{i k a}-i k l(1-a)} .
$$

and the atomic scattering operator $\tau^{(0)}$ (E) given by

$$
\tau^{(0)}(E)=-\frac{2 \Delta \gamma}{2 \gamma-i \Delta} .
$$

where $\gamma=\sqrt{E}$. In terms of the above,

$$
\Sigma(k, E)=\frac{\tau^{(0)}(E)}{l}\left[1-\tau^{(0)}(E) G_{0}{ }^{n}(k, E)\right]^{-1}, \quad I I \cdot D \cdot 10
$$

where

$$
\begin{array}{r}
G_{0}{ }^{n}(k, E)=\frac{-i}{2 \gamma}\left[g^{-}(\gamma+k)+g^{-}(\gamma-k)\right]-\left[\ell\left(E-k^{2}\right)\right]^{-1} . \\
\text { II.D. } 11
\end{array}
$$

To compare the QCA and the numerical results, the energy scale is chosen such that the energy of the bound state of an isolated delta function is minus one, i.e., $E=-\left(\frac{\Delta}{2}\right)^{2}=-1$. In each of the comparisons to be presented, the spectral 
density is obtained by an average over one-hundred chains. The nuber of atoms in each chain was either 500 or 1000 depending upon the level of disorder involved. It is an unfortunate consequence of the numerical wethod tnat the chain length wich can be used is limited by the exponential growth in anplitude of $(x ; E)$ with increasing $x$. This feature places an effective linit on the level of disorder available to investigation.

As mentioned in Chapter I, the integrated density of states $n(E)$ obtained from the QCA gave very good agreement with exact results for positive energies. In Figure 2 is shown a comparison of the $Q C A$ and exact spectral densities for $E=.50$ and two values of $a$. The small arrows indicate the positions of the delta functions for an ordered system $(a=1)$ with lattice spacing $l=10$. The positions are detarmined from the Kronig-Penney relation

$$
\cos (k \ell)=\cos (\gamma \ell)-\frac{\Delta}{2} \frac{\sin (\gamma \ell)}{\gamma}, \quad \text { II.D.12 }
$$

where $\Delta=2.0$. For the disordered system, Figure 2 shows a broadening in response to descreasing $\alpha$ and a diminishing (at $a=.91$ ) and vanishing (at $\alpha=.50$ ) strength of the first two peaks in $A(K, E)$. The $Q C A$ appears to give a very reasonable description of this behavior, although there are slight differences when compared to the numerical results, 
CRIm-DNC 76-7209

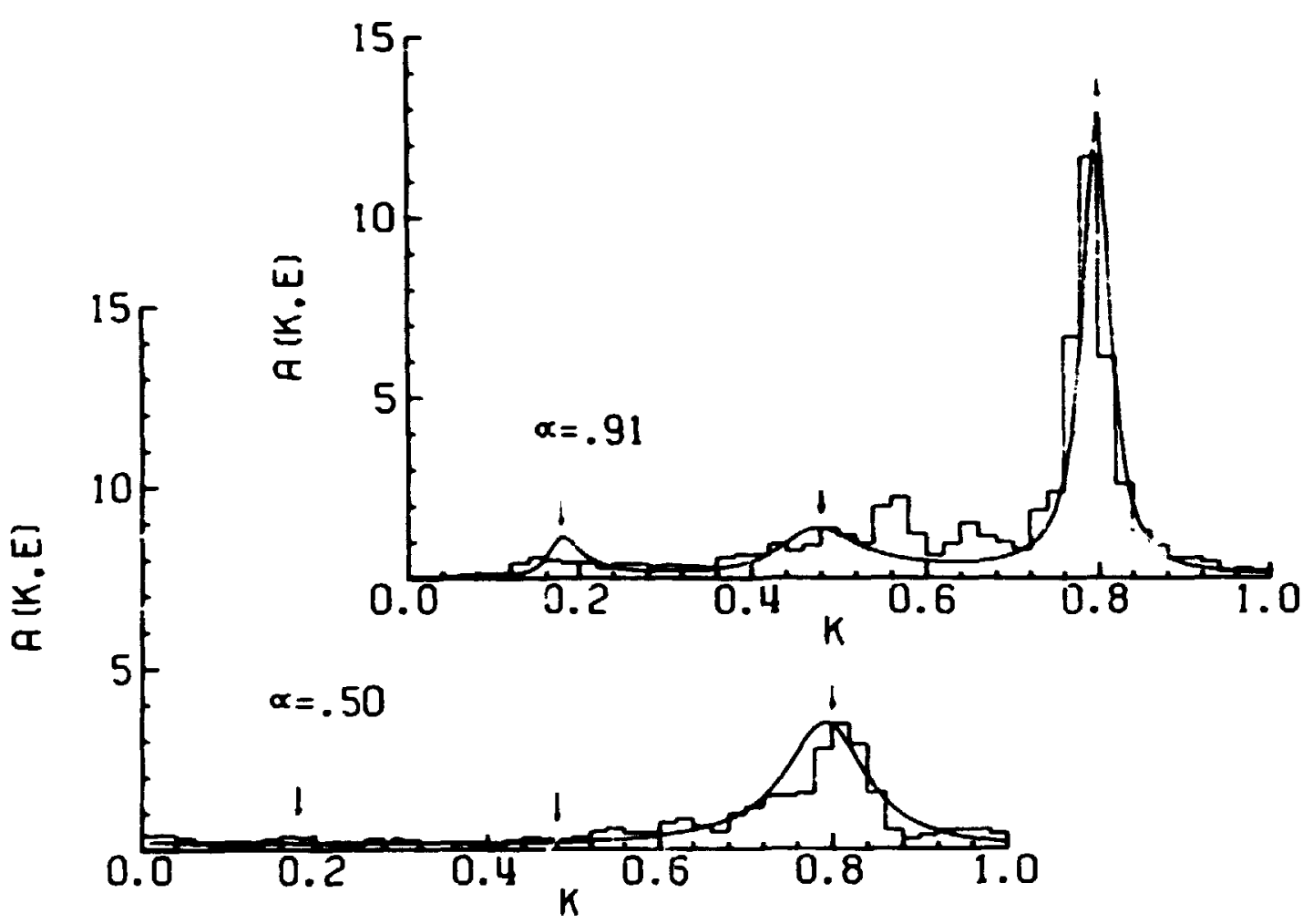

Figure 2. Comparison of OCA (smooth curve) and exact (histogram) spectral densities $A(k, E)$ for $G=.50$ (foreground) and $\alpha=.91$ (background) and energy $E=.50$. Arrows indicate positions of ordered-system $\delta$-functions. 
particularly near $k=.60$ for $\alpha=.91$. At higher energies. the agreement is even better as shown in Figure 3 for $a=.91$ and $E=5.0$.

Turning now to the negative enersy behavior, the results of the numerical calculations are shown in figure 4 for $E=-1.0$ and $a=.91$. One finds broadened peaks in $A(k, E)$ near the k-values determined from Eq. II.D.12. It can be shown (see Appendix C) that within the framework of the QCA the self-energy is a real quanti^y for all energies independent of $a$ and consequently $A(K, E)$ for constant $E$ is a series of delta functions. As observed from Figure 4, A(k,E) is noticeably broadened indicating the failure of the QCA to provide an adequate description at negative energies. The QCA does predict the positions of the peaks show in Figure 4 and is therefore not. without merit. Unfortunately, we cannot test the QCA at smaller values of a for the reasons mentioned earlier.

In summary, this study indicates that the QCA does in fact predict the essential features of the positive energy behavior of the spectral density as well as the integrated density of states. At negative energies, as far as this study can dftermine, the $Q C A$ is able to predict the positions of the peaks in $A(k, E)$, but allows no broadening in response to structural disorder. Continued work is needed to remedy the problems of the QCA at negative energies, but the QCA remains a rather good first approximation. 


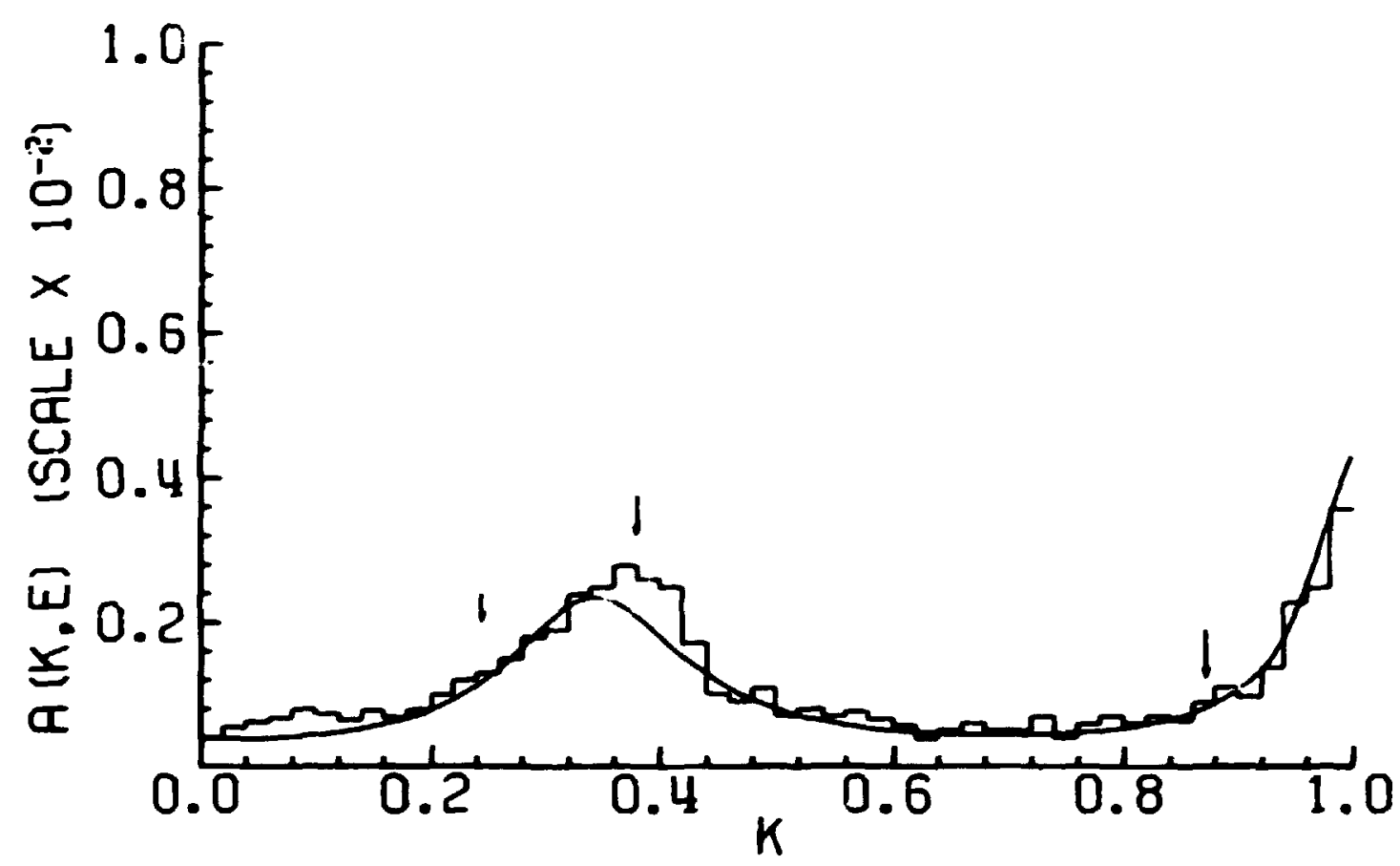

Figure 3. Comparison of $Q C A$ (smooth curve) and exact (histogram) spectral densities $A(k, E)$ for $\alpha=.91$ and energy $E=5.0$. Arrows indicate positions of ordered system $\delta$-functions. 
ORML-OWG 76-7208

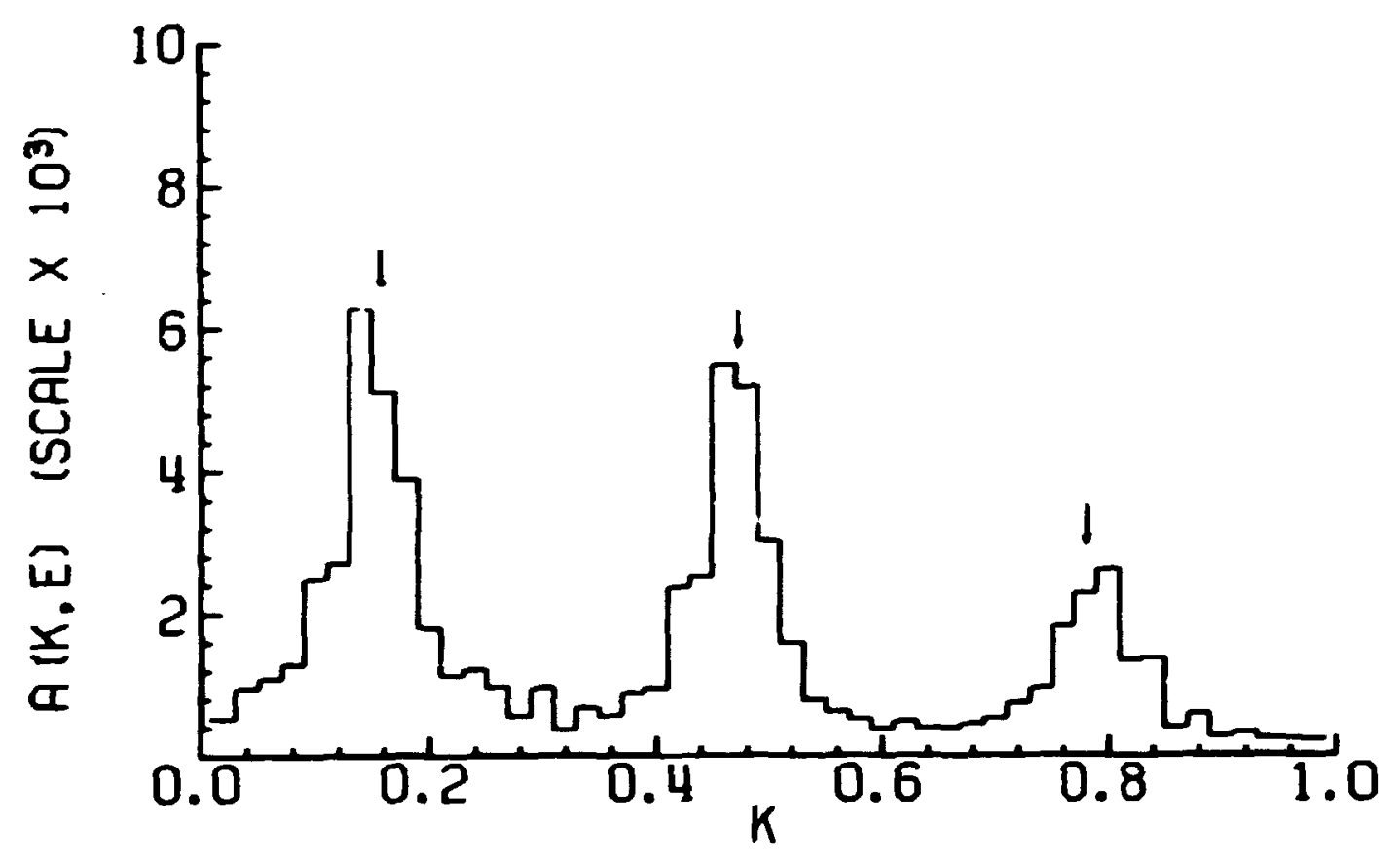

Figure 4. Exact spectral density $A(k, E)$ for $a=.91$ and $E=-1.0$. Arrows indicate positions of ordered-system $\delta$-functions. 


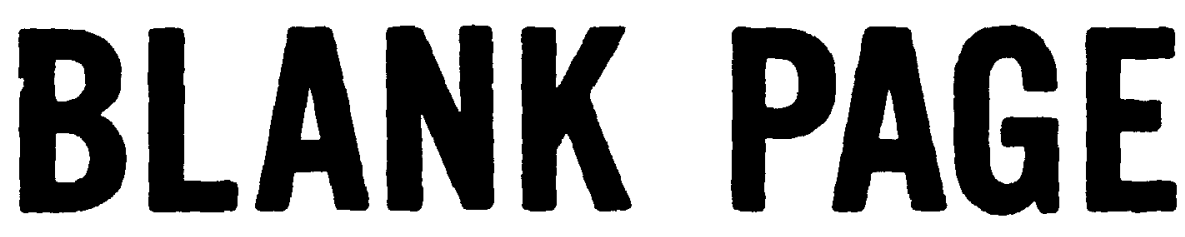


CHAPTER III

TIGHT-BINDING SYSTENS: THE EFFECTS

OF SHORT-RANGE ORDER

This chapter presents a study of the effects of short-range order upon the spectral density function for excitations described by a tight-binding form of Hamiltonian. The scheme developed in Chapter II is applied to the harmonic oscillator Hamiltonian appropriate to lattice vibrations and a tight-binding Hamiltonian describing in a simplified way either electrons or spin waves. Both are studied for nearest-neighbor interactions. Part $A$ describes the details of the calculation for the above Hamiltonians, Part $B$ presents the results of the calculations, and Part $C$ discusses the application of those results to some recent neutron scattering measurements on an amorphous system.

\section{A. The Spectral Density}

The one-dimensional model used in this chapter is the hard-rod model described in chapter II. The first Hamiltnnian to be considered is the harmonic oscillator Hamiltonian

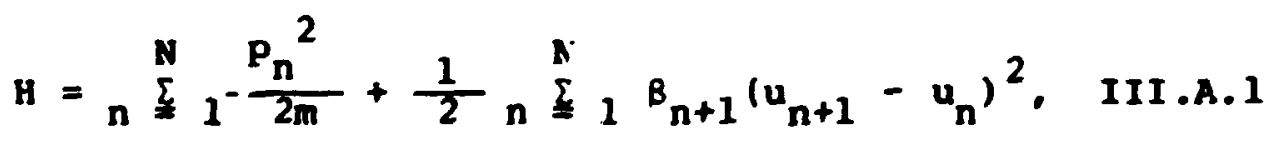


where $p_{n}$ and $u_{n}$ are the momentum and displacement operators for the atom of mass (hereafter set to unity) on site $n$. and $\theta_{n+1}$ is the spring constant which couples sites $n+1$ and n. The second is a tight-binding hamiltonian

$$
H \psi(n)=K_{n} \psi(n)+M_{n} \psi(n-1)+M_{n+1} \psi(n+1) \cdot \operatorname{III.A.2}
$$

$\downarrow(n)$ gives the probability amplitude for finding an excitation at site $n$, and $K_{n}$ and $M_{n}$ are the diagonal and off-diagonal matrix elements, respectively. The calculation of the spectral density for the two Hamiltonians are very s.milar. Details of the calculation will be given only for the tight-binding Hamiltonian, Eq. III.A.2, to be followed by a discussion of the differences that occur for the harmonic oscillator (phonon) system.

The off-diagonal matrix elements $M_{n+1}$ were modeled by an exponential function of the distance $d_{n+1}\left(=x_{n+1}-x_{n}\right)$ between sites $n+1$ and $n$,

$$
M_{n+1}=M_{0} \exp \left\{1-a_{n+1} / \ell\right\} . \quad \text { III.A.3 }
$$

The diagonal matrix elements were set to unity for all $\mathrm{n}$ and the parameter $M_{0}$ was set equal to $-1 / 2$. For an ordered system $(\alpha=1)$ these choices lead to a dispersion relation given by

$$
E(Q)=2 \sin ^{2}(Q / 2)
$$


in units where the average spacing $l$ has been set equal to unity. For the duration of this chapter, the variable $Q$ is used to designate wavevector in order to adopt a notation which will be compatible with that of the experimental results to te discussed in Part $c$ of this chapier.

The spectral density $A(Q, E)$ is defined as

$$
n(Q, E)=\lim _{N \rightarrow \infty} \frac{1}{N}\left\langle\left.\left.\sum\right|_{j=1} ^{n} \exp _{n=1}^{n}\left(-i Q x_{n}\right) \psi_{j}\left(x_{n}\right)\right|^{2} \delta\left(E-E_{j}\right)\right\rangle,
$$

III.A. 5

where $v_{j}$ is the $j^{\text {th }}$ eigenfunction with energy eigenvalue $E_{j}$. As in Chapter II, the calculation of $A(Q, E)$ proceeds from Halperin's (21) reduction of Eq. III.A.5. Actually it will be seen that the treatment presented here is sigh+ly different from Halperin's. Let us define the function

$$
y\left(x_{n} ; E\right)=M_{n} \frac{\phi\left(x_{n-1} ; E\right)}{\phi\left(x_{n} ; E\right)}
$$

where $\left(x_{n} ; E\right)$ is defined to be the unique solution for energy $E$ of the difference equation

$$
M_{n} \phi\left(x_{n-1} ; E\right)+M_{n+1}\left(x_{n+1} ; E\right)+\left(K_{n}-E\right) \phi\left(x_{n} ; E\right)=0
$$

III.A. 7

subject to the conditions 


$$
y\left(x_{1} ; E\right)=Y_{0} \text {. } \quad \text { III.A.8 }
$$

and

$$
y\left(x_{N+1} ; F_{1}\right)=y_{N^{\prime}}
$$

III .A. 9

where $Y_{0}$ and $Y_{N}$ are arbitrary constants. Paralleling the treatment of chapter II, let us define the three functions

$$
\begin{aligned}
& U_{1}\left(x_{n} ; E\right)= \sum_{n}^{n-1} \exp \left(-i Q x_{n} \cdot\right)\left(x_{n} ; E\right) / \exp \left(-i Q x_{n}\right) \phi\left(x_{n} ; E\right), \\
& \text { III.A.10 } \\
& u_{2}\left(x_{n} ; E\right)={ }_{n} \cdot \stackrel{\sum}{=} 1 \phi^{2}\left(x_{n} ; E\right) / \phi^{2}\left(x_{n} ; E\right), \text { III.A.11 }
\end{aligned}
$$

and

$$
U_{3}\left(x_{n} ; E\right)=\frac{\partial}{\partial E} y\left(x_{n} ; E\right)
$$

In terms of these functions, the spectral density can be written as

$A(Q, E)=\lim _{N \rightarrow \infty} \frac{1}{N}\left\langle\frac{\left|U_{1}\left(x_{n+1} ; E\right)\right|^{2}}{U_{2}\left(x_{n+1} ; E\right)}\left|U_{3}\left(x_{n+1} ; E\right)\right| \delta\left(y_{n}-y\left(x_{n+1} ; E\right)\right\} ;\right.$

$$
\text { III.A.13 }
$$

It is straightforward to demonstrate that $U_{2}(x ; E)$ and $U_{3}(x ; E)$ satisfy the equations 


$$
U_{2}\left(x_{n+1} ; E\right)=\frac{y^{2}\left(x_{n+1} ; E\right)}{M_{n+1}^{2}}\left[U_{2}\left(x_{n} ; E\right)+1\right\}, \text { III.A.14 }
$$

and

$$
U_{3}\left(x_{n+1} ; E\right)=\frac{y^{2}\left(x_{n+1} ; E\right)}{M^{2}{ }_{n+1}}\left[U_{3}\left(x_{n} ; E\right)-1\right] . \text { III.A.15 }
$$

from which it follows that

$$
U_{3}\left(x_{n} ; E\right)=-U_{2}\left(x_{n} ; E\right)
$$

Since $U_{2}(x ; E)$ is a positive quantity, $U_{3}(x ; E)$ must be negative. Eq. III.A.13 reduces to

$$
A(Q, E)=\lim _{N \rightarrow \infty} \frac{1}{N}\left(\left|U_{1}\left(x_{N+1} ; E\right)\right|^{2} \delta\left\{y_{N}-y\left(x_{N+1} ; E\right)\right\}\right\rangle
$$

III.A. 17

As in Chapter II, this expression is further reduced by dispensing with the delta function for a long system to obtain

$$
A(Q, E)=\lim _{N \rightarrow 0} \frac{1}{N}\left\langle\left|v_{1}\left(x_{N+1} ; E\right)\right|^{2}\right\rangle
$$

Along with Eq. III.A.3 and Eq. III.A.4, Eq. III.A.7 is used to generate a sequence of wavefunctions $\phi\left(x_{n} ; E\right)$. These are substituted, in turn, into Eq. III.A.18 to obtain $A(Q, E)$. 
The calculation proceeds in the same way for the phonon Hamiltonian with two exceptions. First, the spectral density is customarily written in terms of the frequency $\omega$ and becomes

$$
A(Q, \omega)=\lim _{N \rightarrow \infty} \frac{2 \omega}{N}\left\langle\left|v_{1}\left(x_{N+1} ; \omega\right)\right|^{2} ; . \quad\right. \text { iII.A.19 }
$$

Secondly, the functions $Q\left(x_{n} ; w\right)$ are solutions of the equation of motion

$$
B_{n+1} \phi\left(x_{n+1} ; \omega\right)+B_{n-1} \phi\left(x_{n-1} ; \omega\right)-\left(2 B_{n}-\omega^{2}\right) \phi\left(x_{n} ; \omega\right)=0 .
$$

The spring constants were modeled by an exponential function

$$
B_{n+1}=B_{0} \exp \left\{1-d_{n+1} / l\right\} \text {. }
$$

similar to the matrix elewents for the tight-binding system. The constant $B_{0}$ was set equal to uniry. This choice leads to the ordered-system dispersion relation

$$
\omega(Q)=2\lceil\sin (Q / 2) !
$$

The convergence properties of the spectral density were thoroughly discussed in Chapter II and the arguments will not be repeated here. In the results to be shown, $f_{N}(Q, E)$ was caiculated for each of one-hundred chains and the results 
averaged to given $A(Q, E)$. The number of atoms in a single chain was in all cases $N=500$.

\section{B. Results of the Calculation}

For an ordered system, the spectral density for constant energy consists of a series of delta functions located at the values $Q=Q(w)$ satisfying the appropriate dispersion relation. As discussed in Chapter $I \pi$, these delta functions broaden as the system disorders. In Figure 5 is shorr the phonon spectral density $A(Q, \omega)$ for $a=.75$ and $\omega=.50$. The pos: ions of the ordered-system delta functions are obtained from Eq. III.A.22 and are indicated by arrows in the figure. Long wavelength excitations are rather insensitive to short-range (local) disorder as evidenced by the sharpness of the peak in $A(Q, \omega)$ near $Q=0$. In what would be the second zone for ar, ordered system, $A(Q, \omega)$ is no longer distincuishable as two separate peaks and appears as a single broad structure, shifted toward higher $Q$ from the ordered-system positions. Clearly, the effects of reducing a from unity are most pronounced in the "second-zone." Por the remainder of the results to be presented in this chapter, the peak in the spectral density in the "first zone" will be omitted. The tight-binding results will be discussed first. followed by those for the phonon system. 


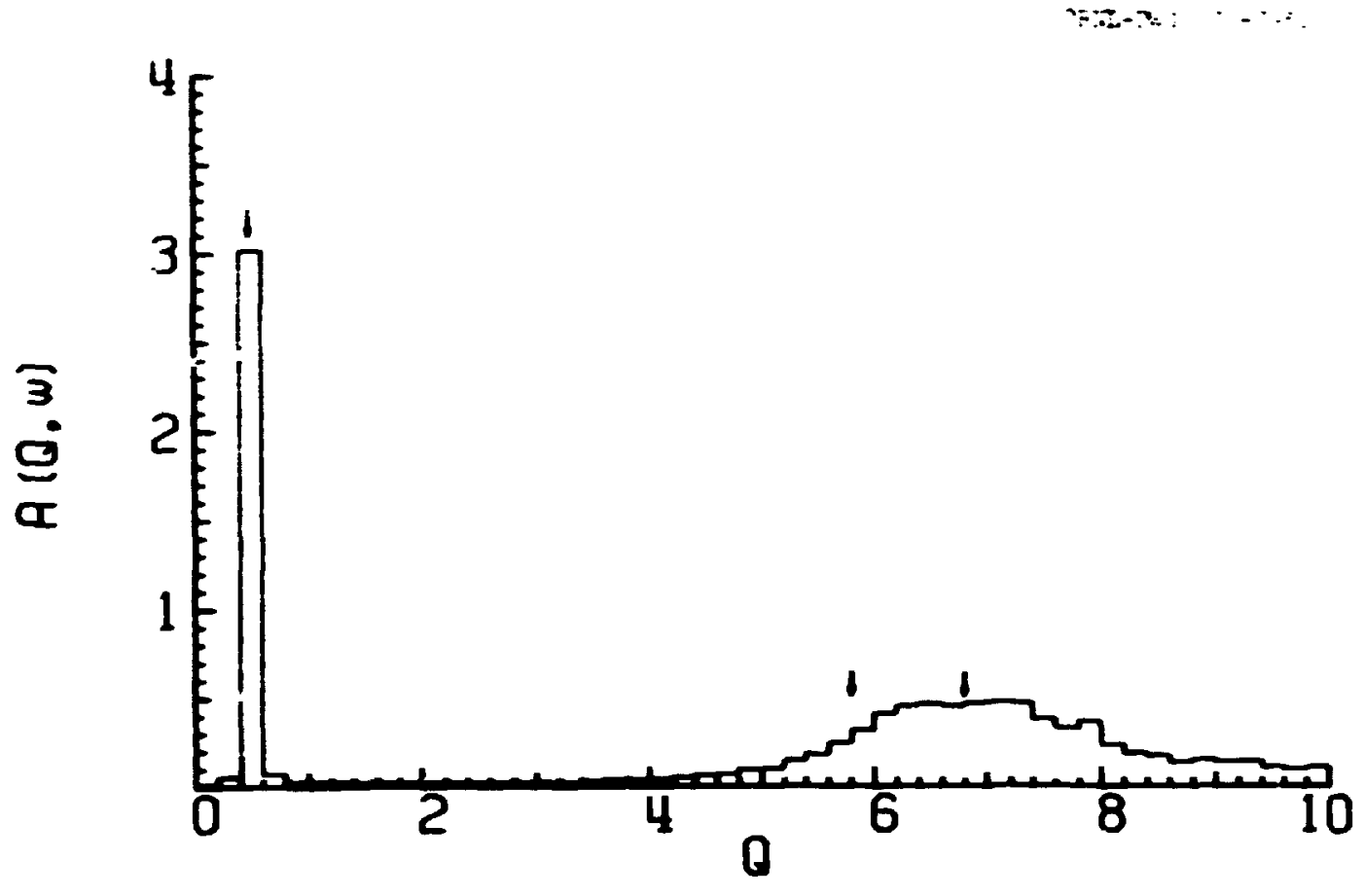

Pigure 5. Spectral density $A(Q, \omega)$ as a function of $Q$ for $w=.50$ and $a=.75$ for the vibrational systen. Arrows indicate positions of ordered-system s-functions. 
Plotted in Figure 6 are the tight-binding system spectral densities for $a=.85$ and four (fixed) energies. The features to be noted in Figure 6 are: (1) that the peaks in $A(Q, E)$ are broadened from the delta functions obtained for an ordered system, (2) that the peaks in $A(Q, E)$ are located near the ordered system peaks (indicated by arrows), and (3) that the strength of the peaks is smallest for low energy. The dominant effect of short-range order is to produce a broadened and slightly shifted version of the ordered-system dispersion. Upon decreasina a to .75 the spectral density takes on the features shown in Figure 7. $A(Q, E)$ is plotted for the sane four energies as in Figure 6 , however, the scale has been reduced by a factor of two relative to Figure $i$. The peaks in $i(Q, E)$ for $a=.75$ are quite broad and are shifted markedly toward higner $Q$. The weighting is such that the mid-band energy $E=1.0$ becomes the strongest feature in the "second zone." The gradual broadening and shifting of the features in $A(Q, E)$ with decreasing a is illustrated in Pigure 8, where the spectral density is plotted as a function of $Q$ for $E=.50$ and three values of $a$. At $a=.50$, nothing remains of the ordered system behavior.

The phonon spectral density $A(Q, \omega)$ is shown in figure 9 for four values of $w$ and $a=.85$. Again, the arrows indisate the positions of the ordered-system delta functions 


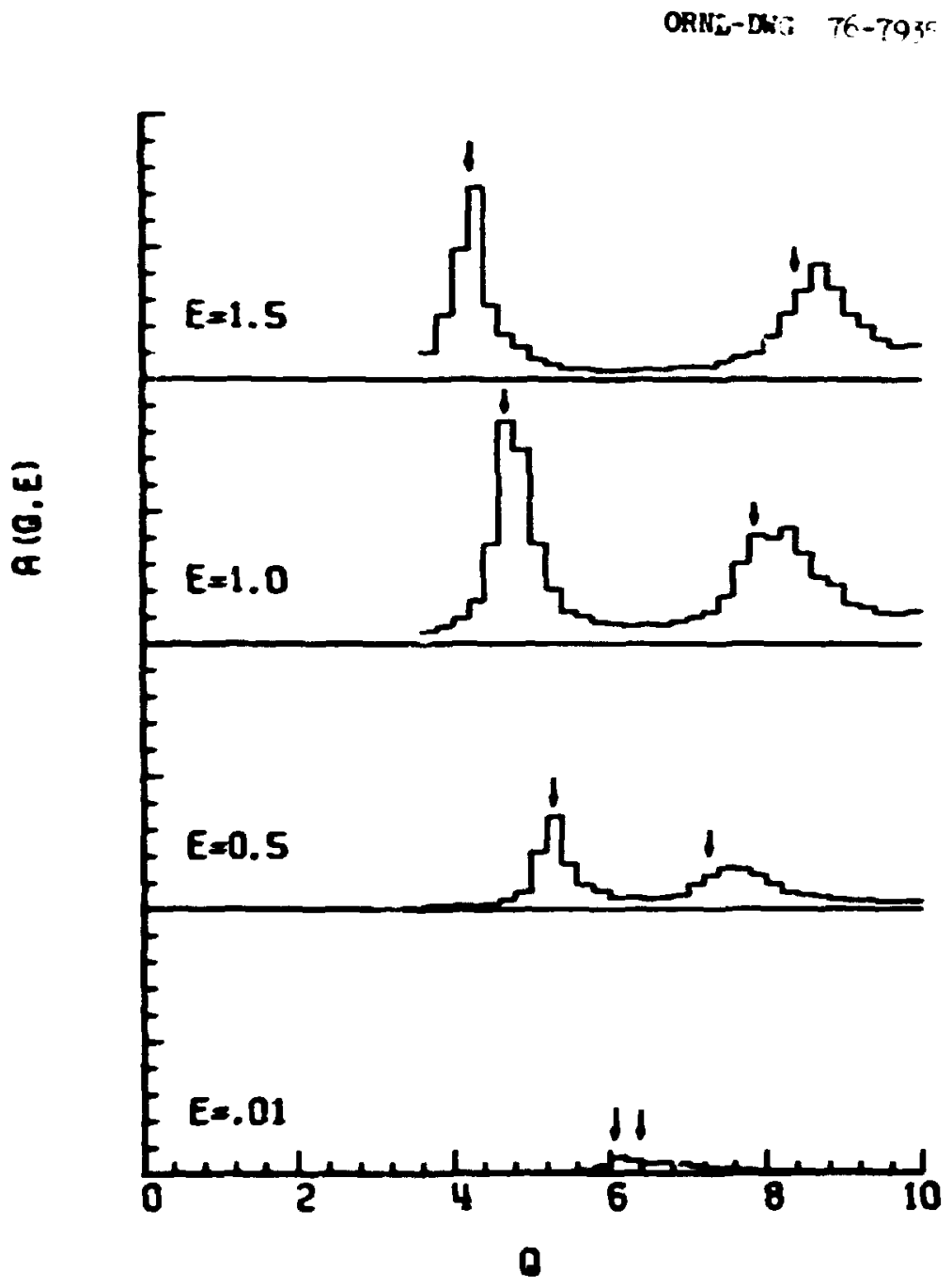

Figure 6. Spectral density $A(Q, E)$ as a function of $Q$ for $x=.85$ and four values of $E$ for the tight-binding system. Arrows indicate ordered-system $\delta$-functions. 


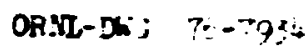

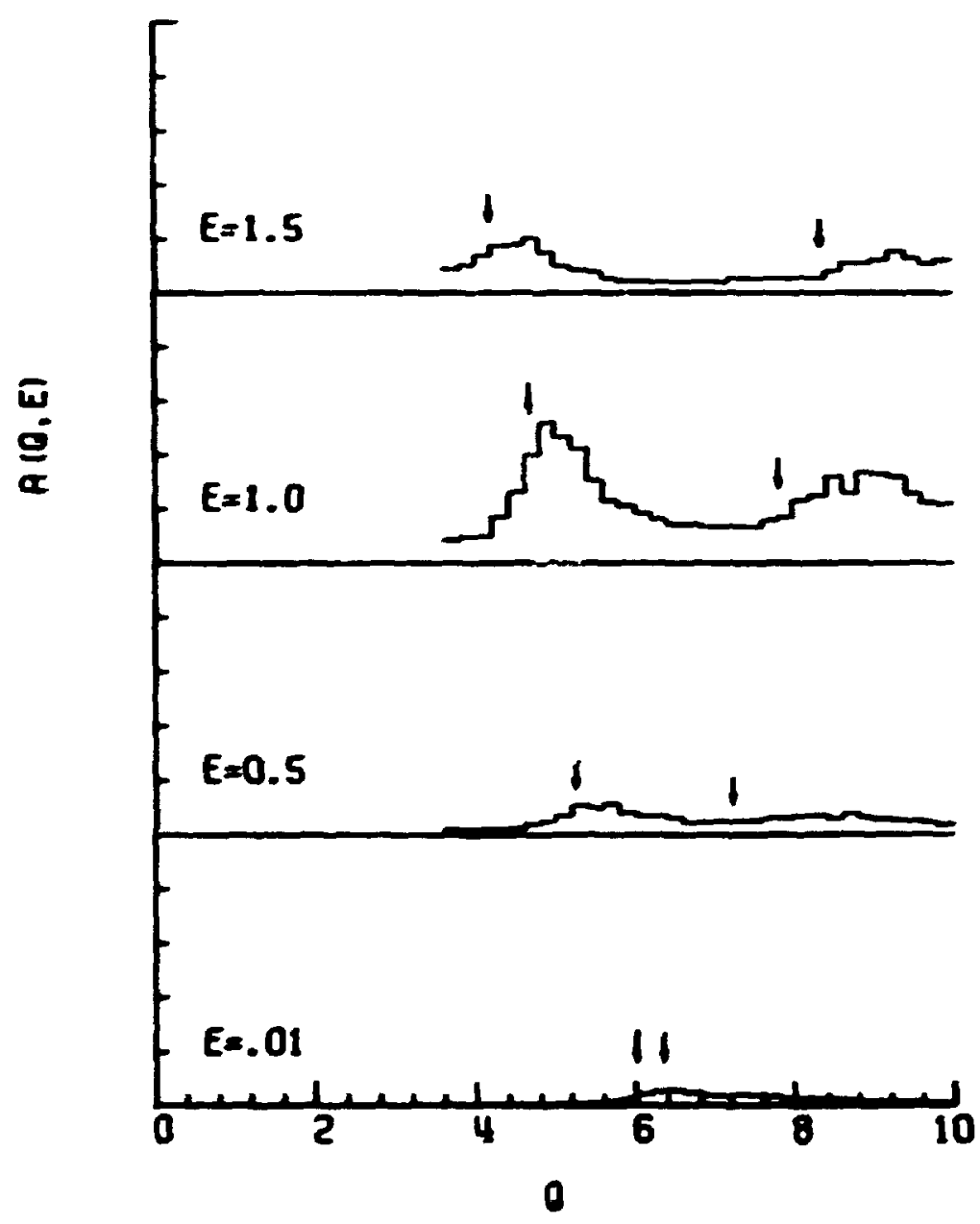

Figure 7, Spectral density $A(Q, E)$ as a function of $Q$ for $a=.75$ and four values of $E$ for the tight-binding system. Arrows indicate positions of ordered-system $\delta$-functions. 


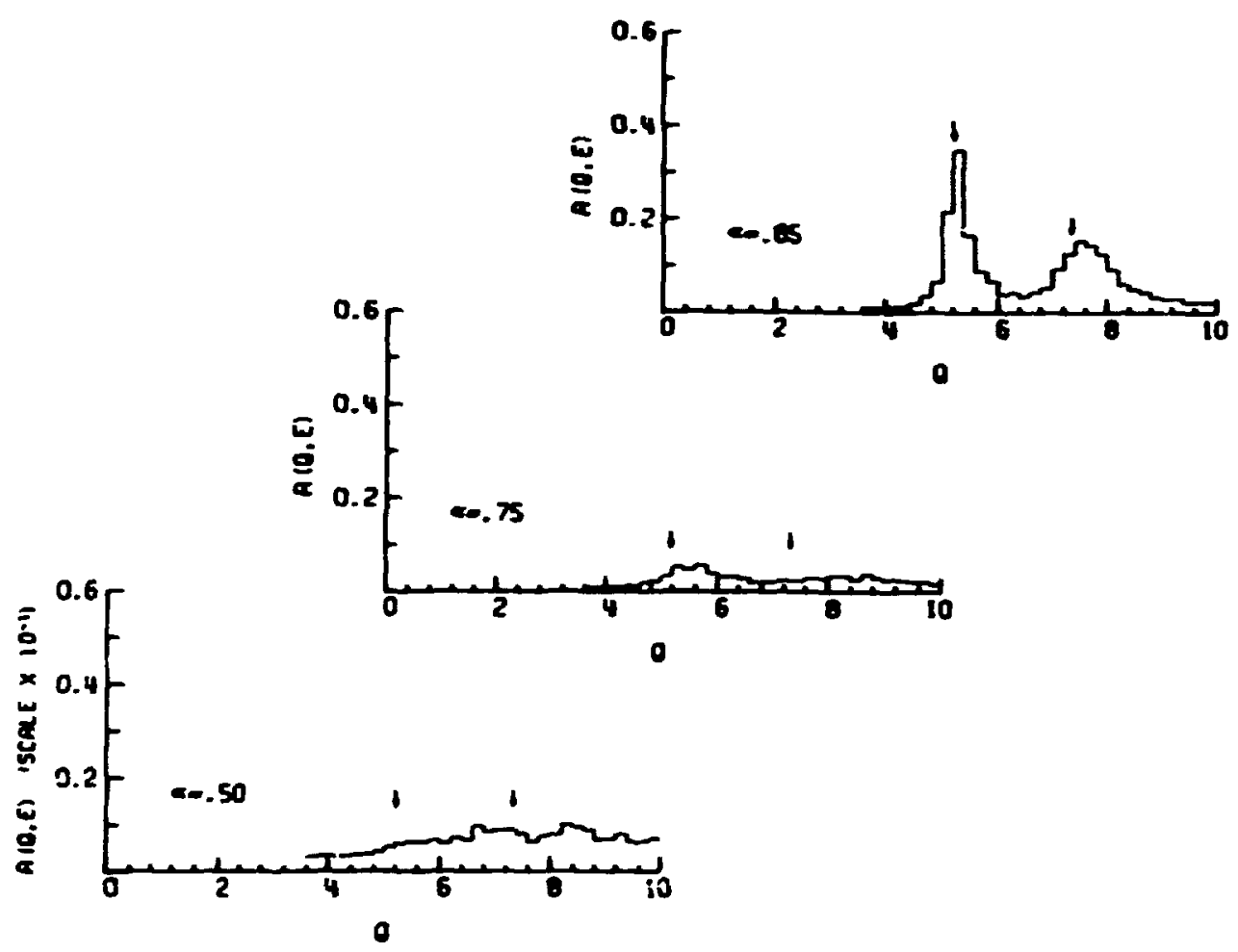

Figure 8. Spectral density $A(Q, B)$ as a function of $Q$ for $E=.50$ and three values of a for the tight-binding system. Arrows indicate positions of ordered-system $\delta$ functions. 


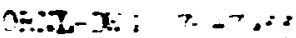

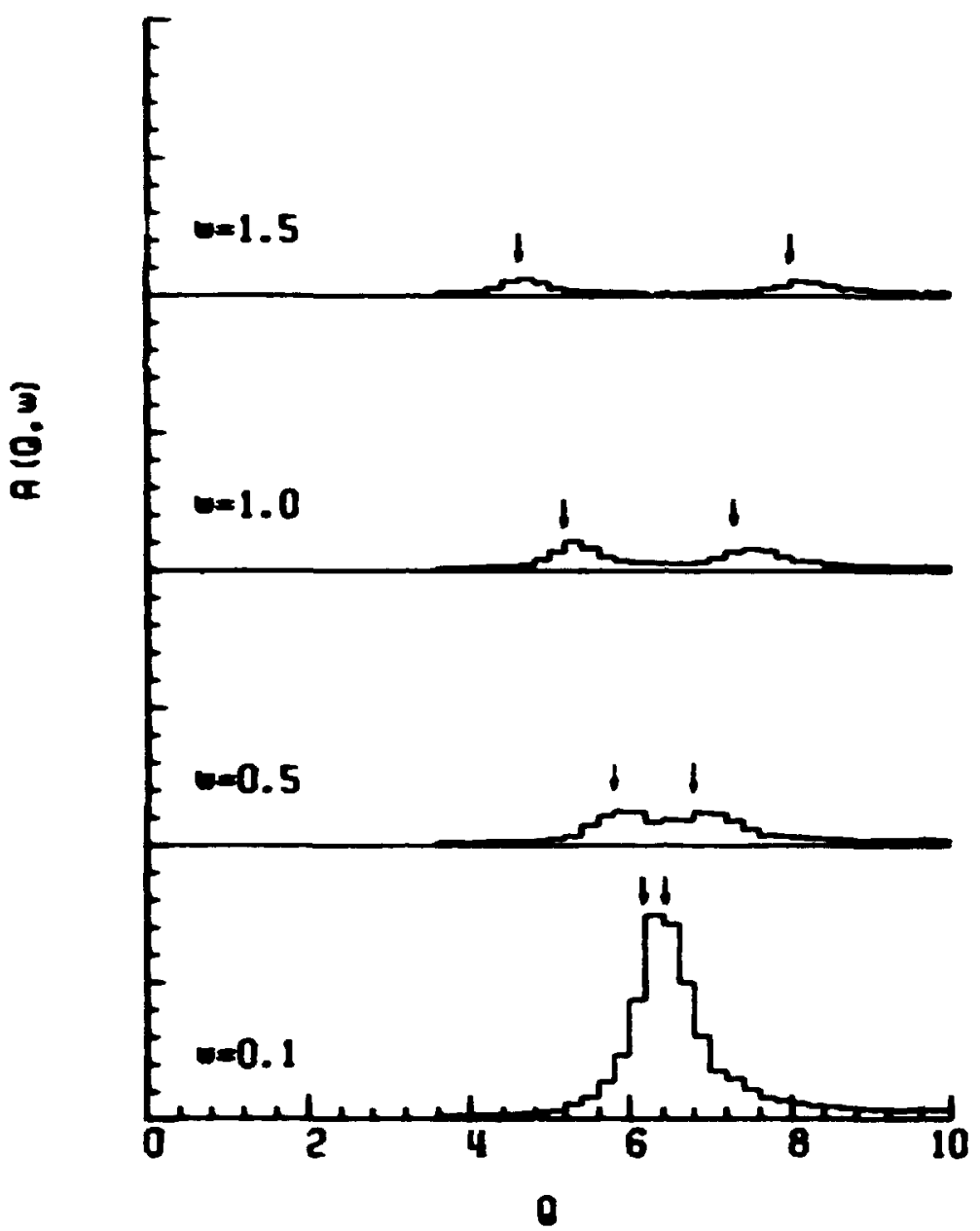

Figure 9. Spectral density $A(0, \omega)$ as a function of $Q$ for $a=.85$ and four values of $\omega$ for the vibrational system. Arrows indicate positions of ordered-system $\delta$-functions. 
as determined from Eq. III.A.22. As with the tight-binding results, the peaks in $A(Q, w)$ are shifted toward $Q$ and are brcadened. The weighting at 10 energy is quite different from that of the tight-tinding systen. The strong-weighting can be understood for noting that, for an ordered system (29)

$$
A(0, \omega)=\frac{1}{2 \omega}[\delta\{\omega-\omega(0)\}-\delta\{\omega+\omega(0)\}] . \quad \text { III.B. }
$$

For $a=.85$, the rodel system is not far from the orderedsystem limit $\alpha=1$. Unless the states mo.ve drastically in $w$ - Q space when a is decreased slightly from urity, the presence of the factor of $\omega^{-1}$ in Eq. III.B.I requires that the strongest weighting occur for $\omega \rightarrow 0$. Plotted in Figure 10 are the phonon spectral densities for the same four values of fixed with $\alpha=.75$. The scale of Figure 10 has been reduced by a factor of ten relative to that of Figure 9. I.roadening and shifting of the peaks in $A(0, w)$ have continued with $A(Q, \omega)$ for $\omega \leq .50$ no longer distinguishable as two distinct peaks. In addition, $A(0, \omega)$ for $\omega=.10$ is no longer more strongly weighted than that for $w=.50$. Pigures 11 and 12 point out more clearly the broadening and shifting of $A(Q, \omega)$ with decreasing a for $\omega=.10$ and $\omega=.50$, respectively. In both cases, all vestiges of the orderedsystem peaks in the spectral density have vanished for $a=.50$. 


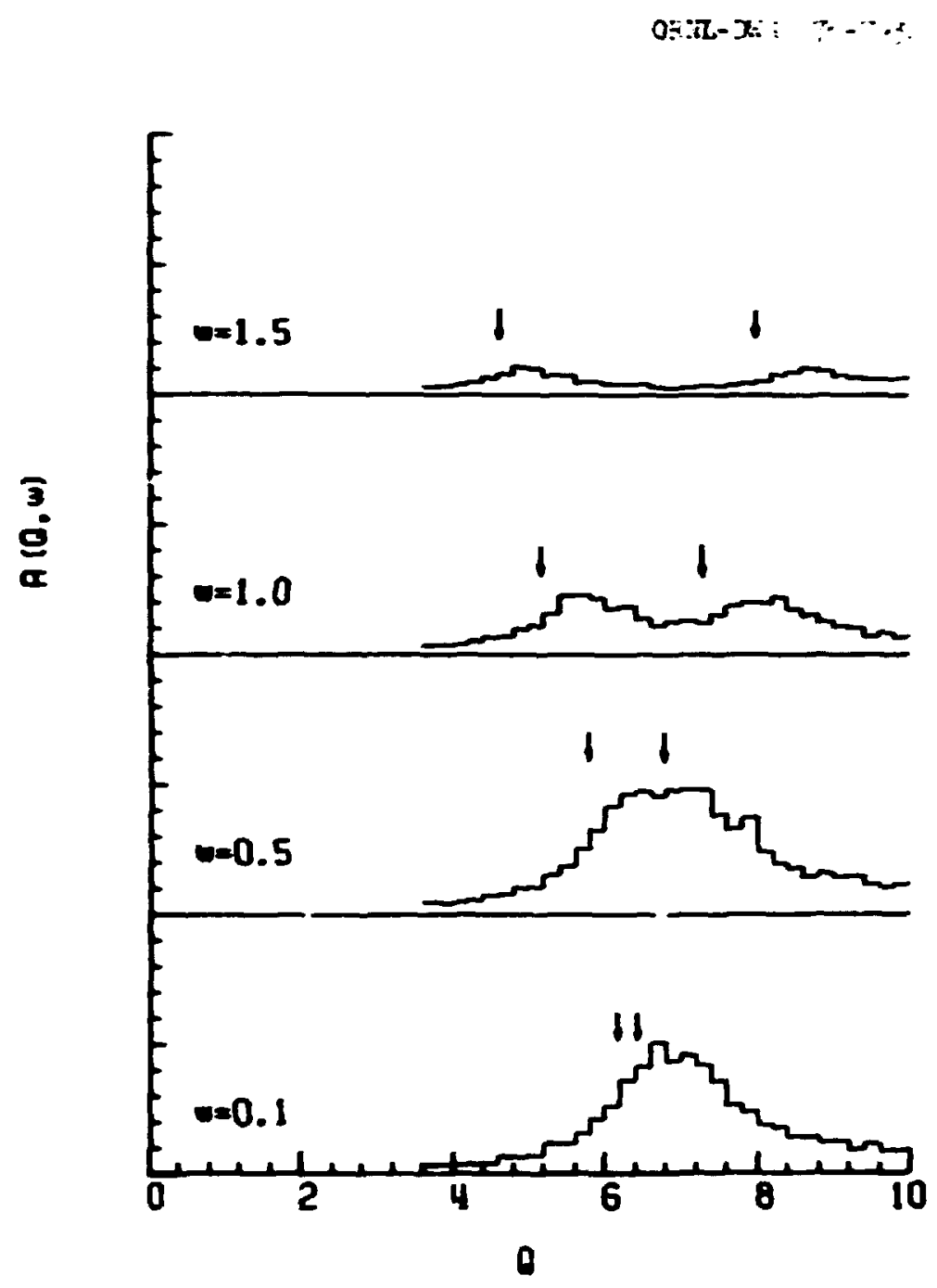

Figure 10. Spectral density $A(0, \omega)$ as a function of $Q$ for $a=.75$ and four values of $w$ for the vibrational system. Arrows indicate positions of ordered-system $\delta$-functions. 


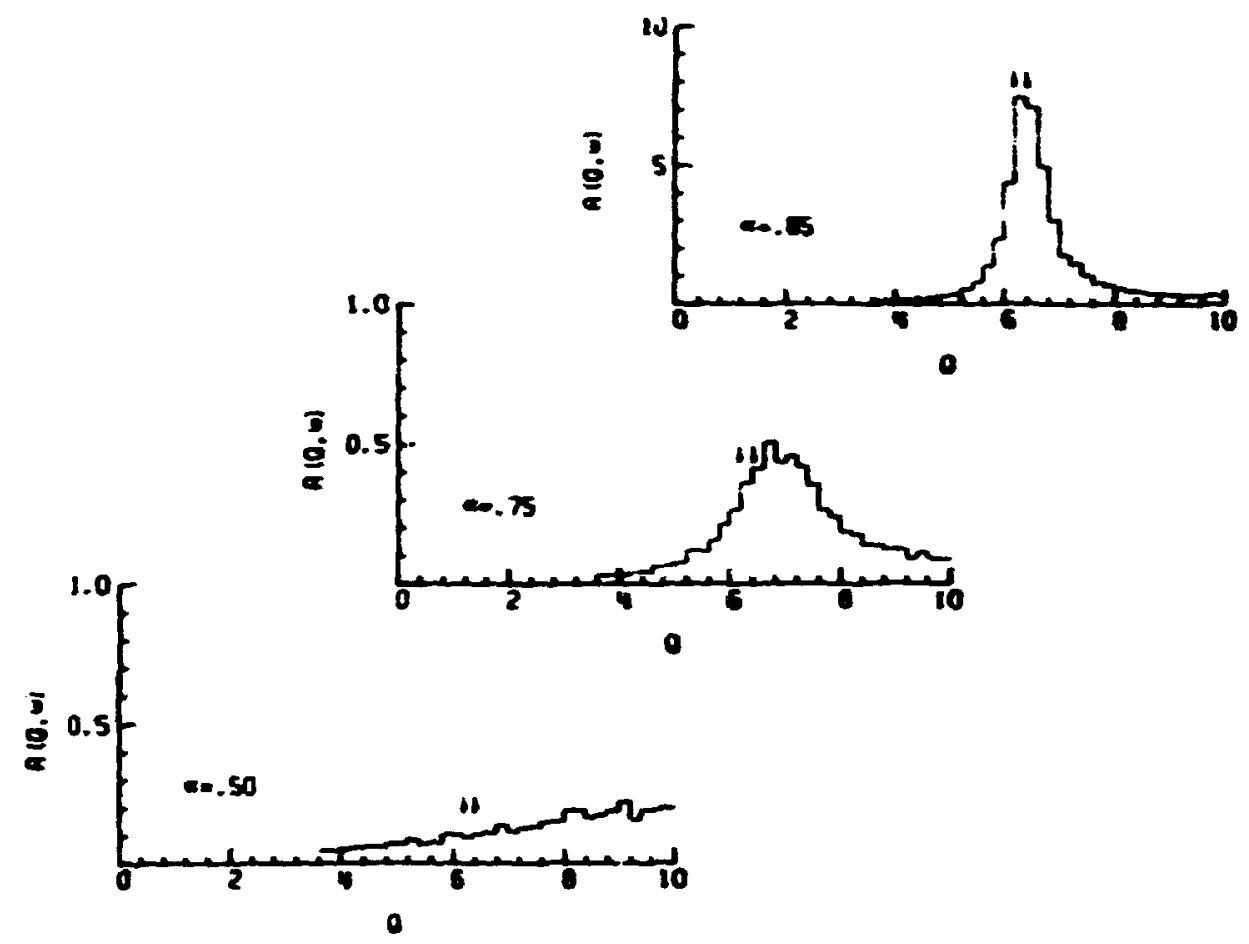

Figure 11. Spectral density $A(Q, i)$ as a function of $Q$ for $\omega=.10$ and three values of $a$ for the vibrational systea. Arrows indicate positions of ordered-system 8 -functions. 
$x-3 x+3 x+y$

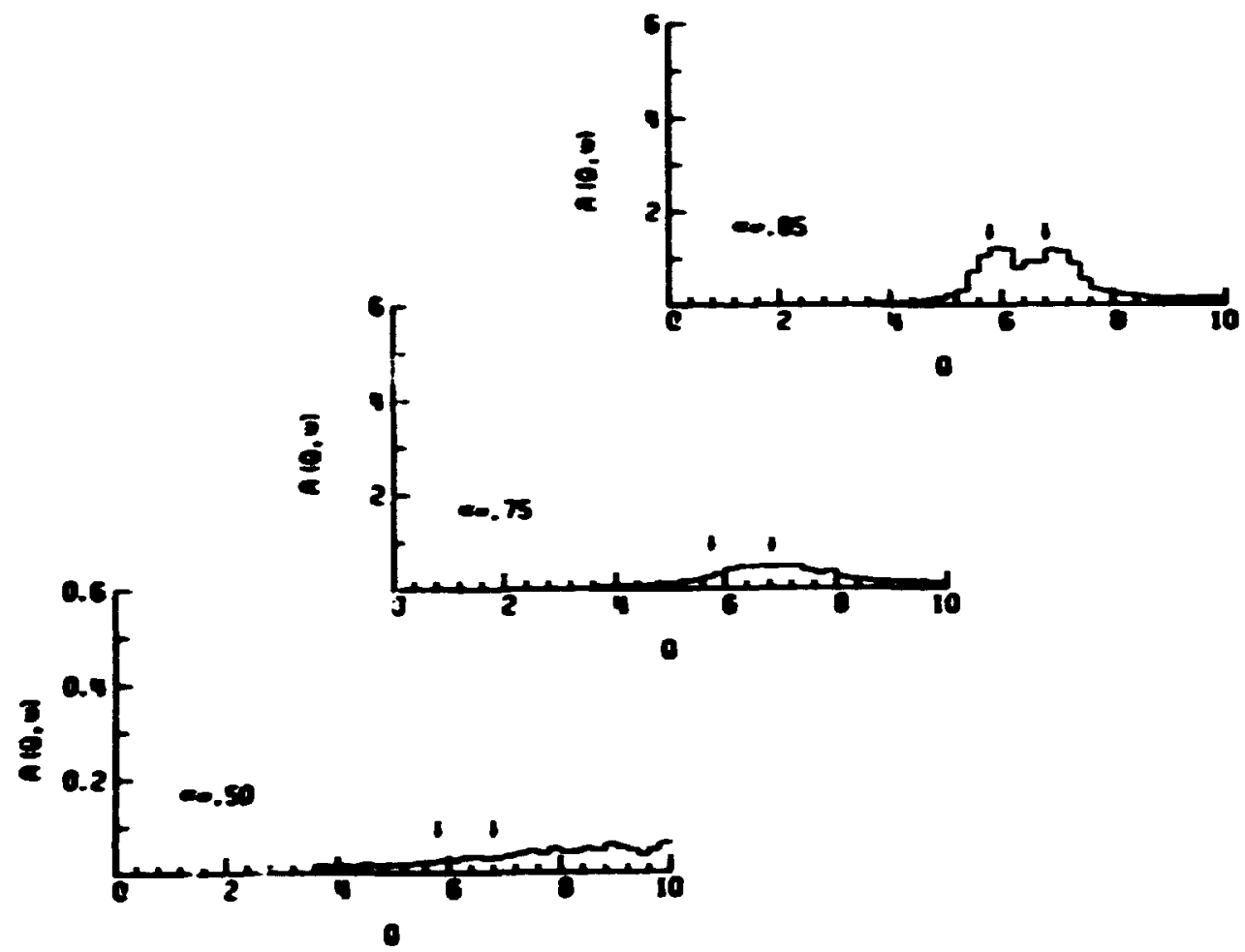

Pigure 12. Spectral density $A(Q, w)$ as a function of $Q$ for $\omega=$.jo and three values of $a$ for the vibrational system. Arrows indicate positions of ordered-system 8 -functions. 


\section{Relation to Experiment}

The results of Part B of this chapter can be used to gain insight into the general features of excitations in three-dimensional systems. The structure in the spectral density does not contain any syitematic distortion due to the one-dimensionality of the system, such as the logarithnic singularities in the density of states. For the phonon system discussed earlier in this chapter with $a=1$ (perfect urder), the Green's function in one dimension takes the form

$$
G(0, \omega)=\frac{1}{\omega^{2}-\omega^{2}(0)+i n} .
$$

In three dimensions, the Green's function becomes

$$
G^{\alpha B}(\vec{Q}, \omega)=\sum_{\mu=1}^{3}\left\{\frac{\varepsilon^{\alpha}(\mu, \vec{Q}) \varepsilon^{\beta}(\mu, \vec{Q})}{\omega^{2}-\omega_{\mu}^{2}(\vec{Q})+i n}, \quad\right. \text { III.C.2 }
$$

where $\mu$ is a polarization index and $\varepsilon^{\alpha}(\mu, \overrightarrow{0})$ is a normalized eigenvector used to diagonalize the force constant matrix $D_{\hat{Q}}^{a B}$ according to

$$
\sum_{B} D_{0}^{q B} \varepsilon^{B}(\mu, \vec{Q})=D_{Q}^{\psi} \varepsilon^{\alpha}(\mu, \vec{Q}) \text {. }
$$

The quantity $\omega_{\mu}{ }^{2}(\vec{Q})$ is related to $D_{\hat{\Omega}}^{\mu}$ by 


$$
{ }_{w}^{2}(\vec{Q})=\omega_{0}^{2}+D_{Q}^{*}
$$

with wo "bare" Einstein phonon frequency. The Green's functions in one and three dimensions have essentially the same form with the single difference that the latter contains a sum over polarizations. The spectral densities sbtained from both Green's functions can be put in a form sirilar to Eq. III B.l containing delta functions with a weighting factor of $\mathrm{w}^{-1}$. The spectral density demonstrates the changes in the one-particle states of the model system in response to structural disorder and the general features can be expected to carry over into three dimensions.

Fiọure 13 shows some recent neutron scattering measurements on the amorphous ferromagnet $\mathrm{Co}_{4} \mathrm{P}$ published by Mook, Hakabayashi, and Pan (30). Shown in Figure 13 are plots for six energies of the neutron inelastic scattering intensity as a function of $Q$. Mbove some energy $E_{1}$, one observes a small peak centered between two larger ones. With decreasino energy the pattern becomes double-peaked and at snme energy $E_{0}$, finally becomes single-peaked. Below $E_{0}$ there exists a small broad structure independent of energy and located near the first peak in the liquid structure factor $s(0)$ (near $Q=3.13 \dot{A}^{-1}$,. In analyzing this data, Mook et al. suggested that if one were to neglect the small central peak at energies above $E_{1}$ and the small structures below $E_{0}$. 

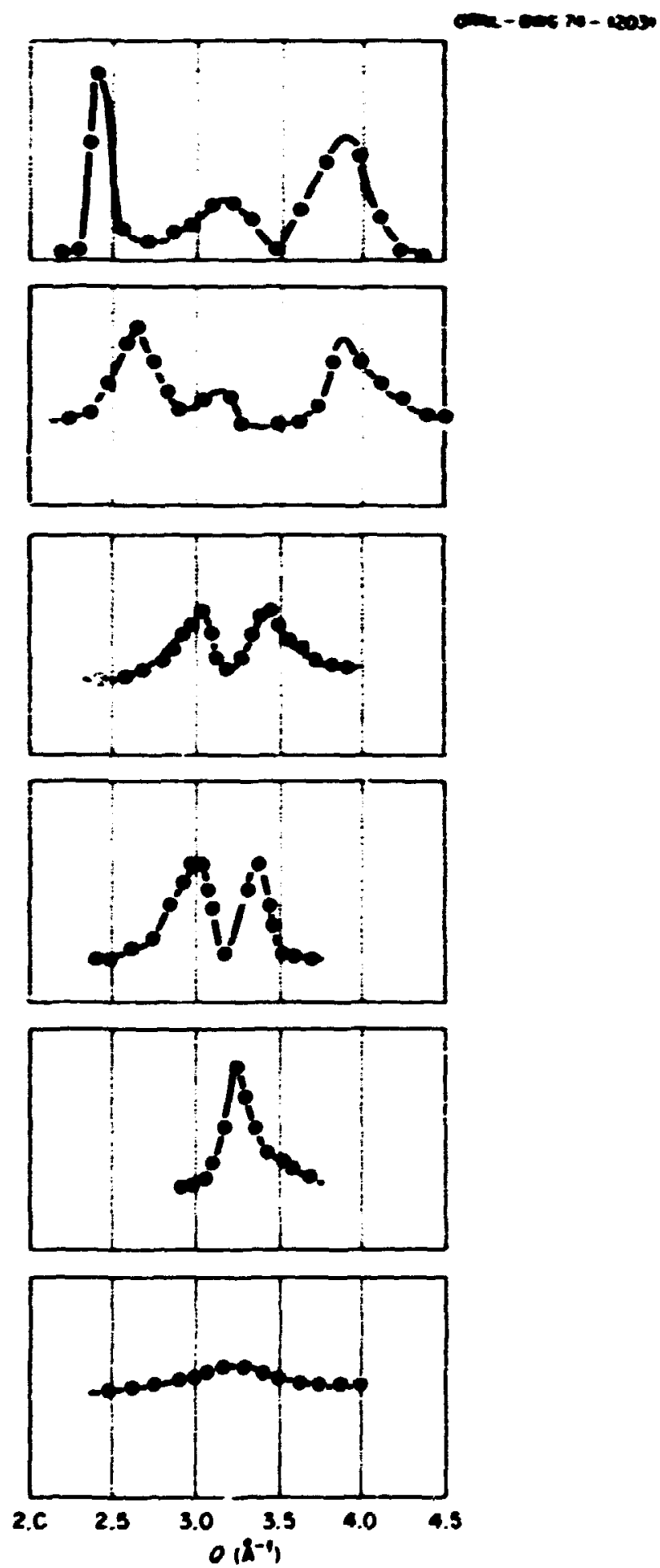

Figure 13. Neutron scattering intensity as a function of $Q$ for several energies (Energy increaser vertically) from Mook et al. (30). 
the peak positions of the reaining features can be plotted as shom in Figure 14. Mis dispersion relation is reminiscent of (30) that observed for rotons (31), suogesting that a new magnetic excitation has been found.

In using the results presented earlier in this chapter to analyze this data, it is useful to establish the role of the spectral dersity in neutron inelastic scattering. The differential cross-section for scattering from lattice vibrations (phonons) is given by

$$
-\frac{d^{2} \sigma}{d \Omega d \omega}=\frac{Q_{2}}{2 ! Q_{1}} s(\vec{Q}, \omega)
$$

where $\vec{Q}_{1}$ and $\vec{Q}_{2}$ are the initial and final momenta of the incident neutron, dâ is an infinitesimal volume element, and $S(\vec{Q}, w)$ is the dynamic form factor. $S(\overrightarrow{0}, w)$ can be written as

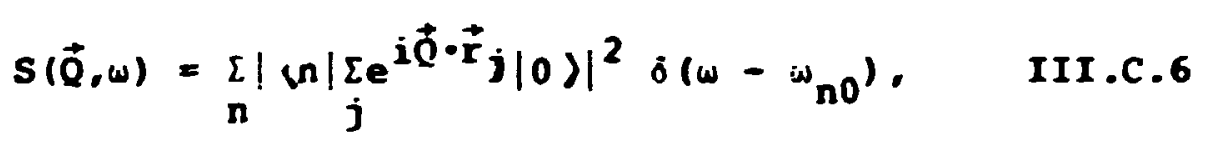

where $|n\rangle$ is an excited state of the system and $|0\rangle$ is the exact ground state. "no is the difference in energy between the ground gtate and the excited state

$$
\omega_{n 0}=E_{n}-E_{0} .
$$

Fxpanding $S(Q, \omega)$ and neglecting those terms describing multiple excitations gives 


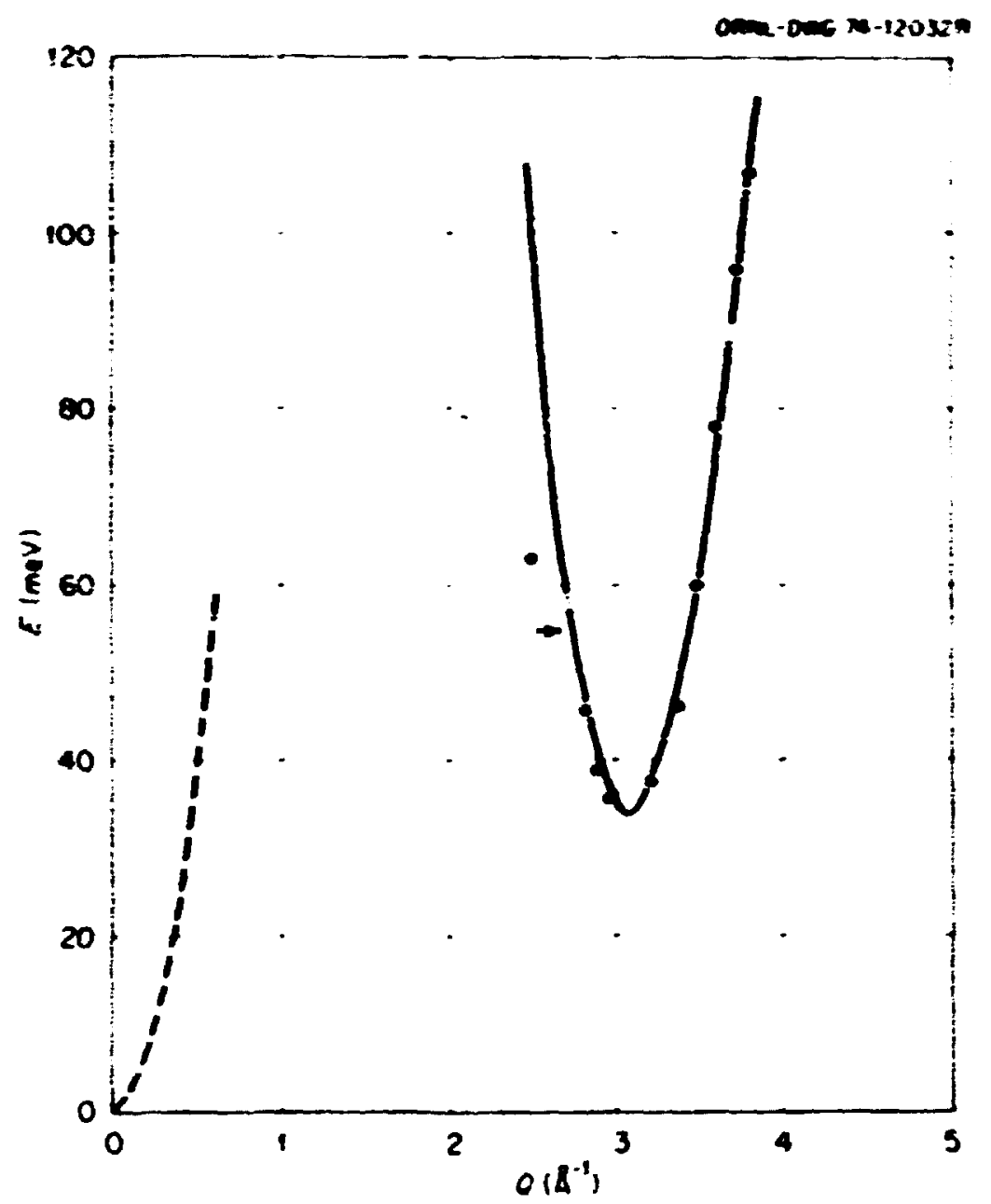

Figure 14. Suggested analysis of data in Pigure 13; from Mook et al. (30). 


$$
S(Q, i)=n e^{-2 N(Q)}\left[I\left(Q j \delta(w)+Q^{2} h(Q, i)\right)\right], \quad \text { III.C.Q }
$$

were I(Q) gives the strength if the elastic scattering contribution to $S(Q, i)$ and $2 w(0)$ is the Debyz-Waller factor. It is clear from Eq. III.C.e that the spectral density $A(Q, w)$ gives the inelastic scattering contribution to $s(0, w)$ from single-particle excitations. For a one-dinensional systen. one carnot actually calculate $s(Q, w)$ or the liquid structure factor $S(0)$ defined as

$$
s(Q)=\frac{1}{H} ; s(0, w) d w .
$$

The Lebye-Waller factor $2 w(Q)$ diverges in one-dimension resulting in $S(Q, \dot{*})=0$ for $a 11 Q$ and $w$. However, this is of no great importance in this work because it is ur.physjcal to scatter from a hypothetical one-dimensional chain. To the extent to which the one-dimensional system used here can se tiken as a model for the effects of short-range order in a solia, the spectrai densities in Figure 5, page 55. Figure 6, page 57, Figure 7, page 58, Fiọure 8, page 59, Figure 9, page 60, Figure 10, page 62, Figure 11, page 63 and Pigure 12, page 64, can be used to interpret the qualitative features of neutron scattering measurements. By comparing Figures 6, 7, 9, and 10 respectively, it emerges as a common feature that the low energy states in a 
tight-binding system do not vanish with the introduction of structural disorder. A Esmparison cî́ Figures 6, page 57 and 13, page 67, makes it appear quite likely that the structure below $E_{0}$ in Figure 13 is real. The calculations indicate that the observations are better explained as a manifestation of the effects of structural disorder upon the ordinary spin waves of the system than as a new type of elementary excitation.

The present calculation also permits a prediction as yet untested by experiment. The results for the vibrational system differ from those of the tight-binding system principally in the weighting of the low energy states. Figures 9 and 10 , pages 60 and 62 , suggest that if an experiment similar to that of Mook et al., were performed on a phonon system, a single peak in the second zone would be found for each energy below a "minimum" energy $F_{0}$, and this peak would be larger than the one that appears in the spin-wave case. 


\section{CHAPTER IV}

\section{SUMMARY}

The preceding chapters have described a numerical investigation of the effects of short-range order nn the spectral density function for a one-dimensional madel $0:$ an amorphous solid. In Chapter II, the calculation was described in detail and applied to a model of an electron in a potential consisting of randomly placed delta functions. The spectral density $A(k, E)$ was found to be given by the mean of the random variable $f_{L}(K, E)$ in the limit $L+\infty$. The convergence properties of $f_{L}(K, E)$ were examined and found to possess the interesting feature that as $L+\infty, f_{2}(k, E)$ is not self-àveraging (ergodic). That is, $f_{L}(K, E)$ does not approach ts mean value with zero variance as $L$ becomes large. It was shown that ... alte rative procedure exists for calculating $A(k, E)$ for a single long chain of atoms, but that method leads to numerical difficulties. The actual determinations of $A(K, E)$ was made by calculating $f_{L}(K, E)$ for several moderately long chains and averaging the results.

The model calculation in Chapter II was compared with $A: k, E)$ obtained in the quasicrystajiline approximation (QCA). The QCA agreed very well with the essentially exact results in the positive energy (weak-scattering) regime, but proved inadequate in the ncyative energy (strong-scattering) regime. 
These conclusions are consistent with earlier results for the integrated density of states.

In Chapter III, the spectral densities associated with a tight-binding Hamiltonian and a phonon Hamiltonian were studied. For both the tight-binding and phonon systems, the dominant effect of short-range order in a structurally disordered model is to produce a broadened and slightly shifted (in $Q$ ) version of the ordered-system spectral density. The most striking difference between the spectral densities for the two systems is the weighting given to low energy states. The model calculations of Chapter III were used to analyze the qualitative features of recent neutron inelastic scattering measurements on an amorphous ferromagnetic system. Based on the calculations, the experimental data support the conclusion that the observed excitations were ordinary spin waves, with properties suitably altered by the structural disorder of the system.

The treatments presented in this work by no means exhaust the possible applications of the numerical method. A natural extension of the work in Chapter II would be a calculation of $A(k, E)$ for a model binary alloy and a comparison with the spectral density obtained from the coherent potential approximation (CPA). The methods of Chapter III could be applied to an investigation of the vibrational properties of a binary alloy. 
LIST OF REFERENCES

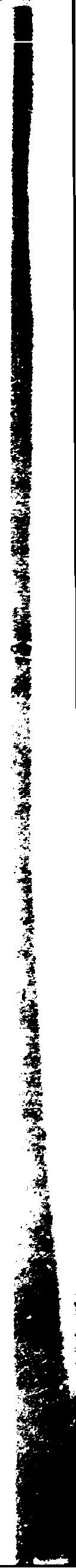




\section{LIST OF REFERENCES}

1. J. S. Faulkner, Int. J. Q. Chem. 5, 543(1971) .

2. P. Soven, Phys. Rev. 156, 809 (1967).

3. B. Velicky, S. Kirpatrick, and H. Ehrenreich, Phys. Rev. 175, 747 (1968).

4. G. M. Stocks, R. W. Williams, and J. S. Faulkner, Phys. Rev. Lett. 26, 253(1971).

5. G. M. Stocks, R. W. Williams, and J. S. Faulkner, Phys. Rev. B4, $4390(1971)$.

6. G. M. Stocks, R. W. Williams, and J. S. Faulkner, J. Phys. F3. $1688(1973)$.

7. S. F. Edwards, Phil. Mag. 6 (Ser. 8), 617(1961).

8. B. G. Nickel and w. H. Butler, Phys. Rev. Lett. 30, $373(1973)$.

9. M. Lax, Rev. Mod. Phys. 23, 287 (1951); Phys. Rey. 85, 621 (1952).

10. L. M. Schwartz and H. Ehrenreich, Ann. Phys. (N.Y.) 64. $100(1971)$.

11. J. S. Faulkner, Phys. Rev. B1, 934 (1970).

12. J. R. Klauder, Ann. Phys. (N.Y.) 14, $43(1961)$.

13. B. L. Gyorffy, Phiys, Rev. B1, 3290 (1970).

14. J. Korringa and R. L. Mills, Phys. Rev. Bㅌ, 1654 (1972).

15. L. M. Roth, Phys. Rev. Bg, 2476(1974).

16. Y. Ishida and F. Yonezawa, Prog. Theor. Phys. 49, $731(1973)$.

17. P. Dean, Rev, Mod, Phys, 44, $127(1972)$.

18. H. K. Peterson, L. M. Schwartz, and W. H. Butler, Phys. Rev. B11, 3678 (1975). 
19. R. Alben, M. Blune, H. Krakaver, and L. Schwartz, Phys. Rev. B12, 4090 (1975).

20. R. Mlben and M. Thorpe, J. Phys. C8, L275 (1975).

21. B. I. Halperin, Phys. Rev. 139. Al04(1965).

22. Z. W. Salzburg, R. W. zuanzig, and J. G. Firkwood, J. Chem. Phys. 21, 1098 (1953).

23. R. E. Borland, Proc. Roy. Soc. 274, 529 (1963).

24. B. I. Halperin, Advan. Chem. Phys, 13, $123(1967)$.

25. W. A. Harrison, Solid State Theory (New York: McGrawHi11, 1970), pp. 228-229.

26. H. M. James and A. S. Ginzbarg, J. Phys. Chem. 57, $840(1953)$.

27. A. A. Sveshnikov, Applied Methods of the Theory of Random Functions (Iondon: Pergamon, 1966).

28. E. Parzen, Stochastic Processes (San Francisco: HoldenDay, 1964), page 116.

29. S. Doniach and E. Sondheimer, Green's Punctions for Solid State Physicists (Reading: Benjamin, 1974). page 43 .

30. H. A. Hook, N. Wakabayashi, and D. Pan, Phys, Rev. Lett. 34. 1029 (1975).

31. D. G. Henshaw and A. D. B. Hoods, Phys. Rev. 121. $1266(1961)$.

32. J. B. Thomas, Applied Probability and Random Processes (New York: Wiley and Sons, 1971). 
APPEMDIX A

DERIVATION OF EQ. II .C. 6

Consider the integral

$$
I=\int_{00}^{L L} f(x-y) d x d y .
$$

A. 1

Insert a delta function integration

$$
I=\int_{00}^{L L} \int_{-L}^{L} \delta\{u-(x-y)\} f(u) d u d x d y .
$$

Rearranging orders of integration and splitting the integration over $u$ into positive and negative contributions yields

$$
\begin{aligned}
I= & \int_{-L}^{0} f(u) \int_{0}^{L}\left[\int_{0}^{L} \delta\{y-(x-u)\} d y\right] d x d u \\
& +\int_{0}^{L} f(u) \int_{0}^{L} \underset{0}{L}[\delta \delta\{y-(x-u)\} d y] d x d u .
\end{aligned}
$$

Substituting $u=-u^{\prime}$ in the first integral gives

$$
\begin{aligned}
I= & \int_{0}^{L} f(-u) \int_{0}^{L}\left[\int_{0}^{L} \delta\{y-(x+u)\} d y\right] d x d u \\
& +\int_{0}^{L} f(u) \int_{0}^{L}\left[\int_{0}^{L} \delta\{y-(x-u)] d y\right] d x d u,
\end{aligned}
$$


where $u$ is always positive. The first integral in Eq. A.4 is nonzero only if $0 \leq x \leq(L-u)$. The second integral is nonzero only if $u \leq x \leq$ L. Eq. A. 4 becomes

$$
\begin{aligned}
I= & \int_{0}^{L} f(-u) \int_{0}^{L-u} \underset{0}{L}[\delta(y-(x+u)\} d y] d x d u \\
& +\int_{0}^{L} f(u) \underset{u}{L} \underset{0}{L} \delta\{y-(x-u)\} d y j d x d u .
\end{aligned}
$$

Perfcrming the integration indicated results in

$$
I=\int_{0}^{L}(L-u)\{f(u)+f(-u)\} d u \text {. A.6 }
$$

Application of Eq. A.6 to Eq. II.C.5 with $f(x-y)=e^{i k(x-y)}$ $R(x-y)$ leads directly to Eq. II.C.6. 


\section{APPENDIX B \\ DE.RIVATION OF EQ. II.C.11}

The reduction of Eq. II.C.lu begins by noting that for normally distributed random variables with zero mean,

$$
\begin{aligned}
& \langle g(w ; E) g(x ; E) g(y ; E) g(z ; E)\rangle=\langle g(w ; F) g(x ; E)\rangle\langle g(Y ; E) g(z ; E) ; \\
& \quad+\langle g(w ; E) g(y ; E ;\rangle\langle g(x ; E) g(z ; E)\rangle \\
& \quad\langle(\sigma ; w ; E) g ; z ; E)\rangle\langle g(x ; E(g(Y ; E)\rangle .
\end{aligned}
$$

This property of normally distributed random variables can be found in standard texts on probabiity (see, for example, reference 32). Substituting Eq. B.1 into Eq. II.C.10 gives

$$
\begin{aligned}
& \left\langle\left\{f_{L}(k, E)\right\}^{2}\right\rangle=\left[\frac{1}{L} \int_{00}^{L L} e^{i k(w-x)}\langle g(w ; E) g(x ; E) d w d x]\right. \\
& {\left[\frac{1}{L} \int_{00}^{L I} \int e^{i k(y-z)}\langle g(y ; E) g(z ; E)\rangle d y d z\right]} \\
& +\frac{1}{L^{2}}\left[\int_{00}^{L L} e^{i k(w-z)} \lg (w ; E) g(z ; E) ; d w d z\right] \\
& {\left[\begin{array}{l}
\ln L \\
\iint_{0} e^{i k(y-x)} \\
00
\end{array}\right.}
\end{aligned}
$$

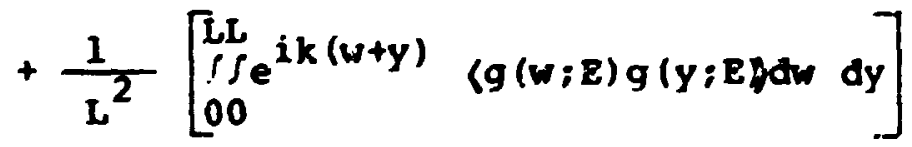




$$
\left[\begin{array}{ll}
L L \\
\iint_{0} e^{-i k(x i z)} & \langle g(x ; E) g(z ; E) ; d x d z
\end{array}\right] \text { B.2 }
$$

The first two terms are easily identified as $\left(f_{L}(k, E)\right)^{2}$ and Eq. B. 3 simplifies to

$$
\begin{aligned}
& \left.\left\langle\left\{f_{L}(k, E)\right\}^{2}\right\rangle=2: f_{L}(k, E)\right\rangle^{2}
\end{aligned}
$$

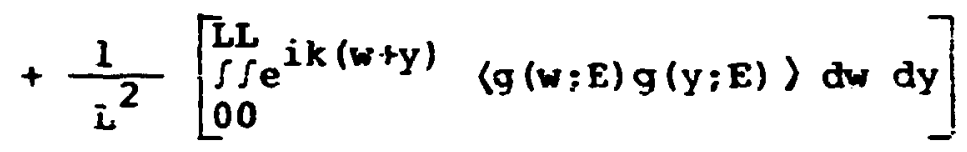

$$
\begin{aligned}
& {\left[\int_{00}^{L L} e^{-i k(x+z)}\langle g(x ; E) g(z ; E)\rangle d x d z\right]}
\end{aligned}
$$

It will now be demonstrated that in the limit $L+\infty$, the first of the two integrals in the second term of the above equation vanishes.

Let $R(w-y)=\langle g(w ; E) g(y ; E)\rangle$ and let us define

$$
J=\frac{1}{L} \int_{00}^{L L} \int^{i k(w+y)} R(w-y) d w d y .
$$

As in Appendix A, introducing a delta function integration puts $J$ in the form

$$
J=\frac{1}{L} \int_{-L 00}^{L L L} \int^{i k(u+2 y)} R(u) \delta\{u-(w-y)\} d w d y d u . B . \Xi
$$


Ferforming exactly the same separation of the integral as that of Appendix $A$ and invoking $R(u)=R(-u)$ gives

$$
J=2 \int_{0}^{L} R(u) e^{i k L}\left[\frac{\sin \{k(L-u)\}}{k L}\right] j u .
$$

Taking the limit of $J$ as $L \rightarrow \infty$ gives zero. 


\section{APPENDIX C}

THE QCA SELF-ENERGY

It will be shown here that the self-energy $\sum(K, E)$ is, for negative energies, a real quantity for the model described in Chapser II. Let us define $k=|E|^{1 / 2}$. For negative energy the scattering operator, is real and given by

$$
\tau^{(0)}(E)=-\frac{2 \Delta K}{2 K-\Delta} \text {. }
$$

It remains to be shown that the quantity $G_{0}{ }^{\prime \prime}(k, E)$ defined in Eq. II.D.11 is real. Replacing $\gamma$ by ik in Eq. II.D.11 shows that $g^{-}(r+k)+g^{-}(\gamma-k)=g^{-}(i k+k)+g^{-}(i k-k)$ is the quantity of interest.

$$
\begin{aligned}
& g^{-}(\gamma+k)+g^{-}(\gamma-k)=\frac{e^{i k a} e^{-K a}}{1-e^{i k a} e^{-K a}-i(i k+k) \ell(1-a)} \\
&+\frac{e^{-i k a} e^{-K a}}{1-e^{-i k a} e^{-K a}-i(i K-k) \ell(1-a)} . \\
& C .2
\end{aligned}
$$

Changing the denominators slightly gives 


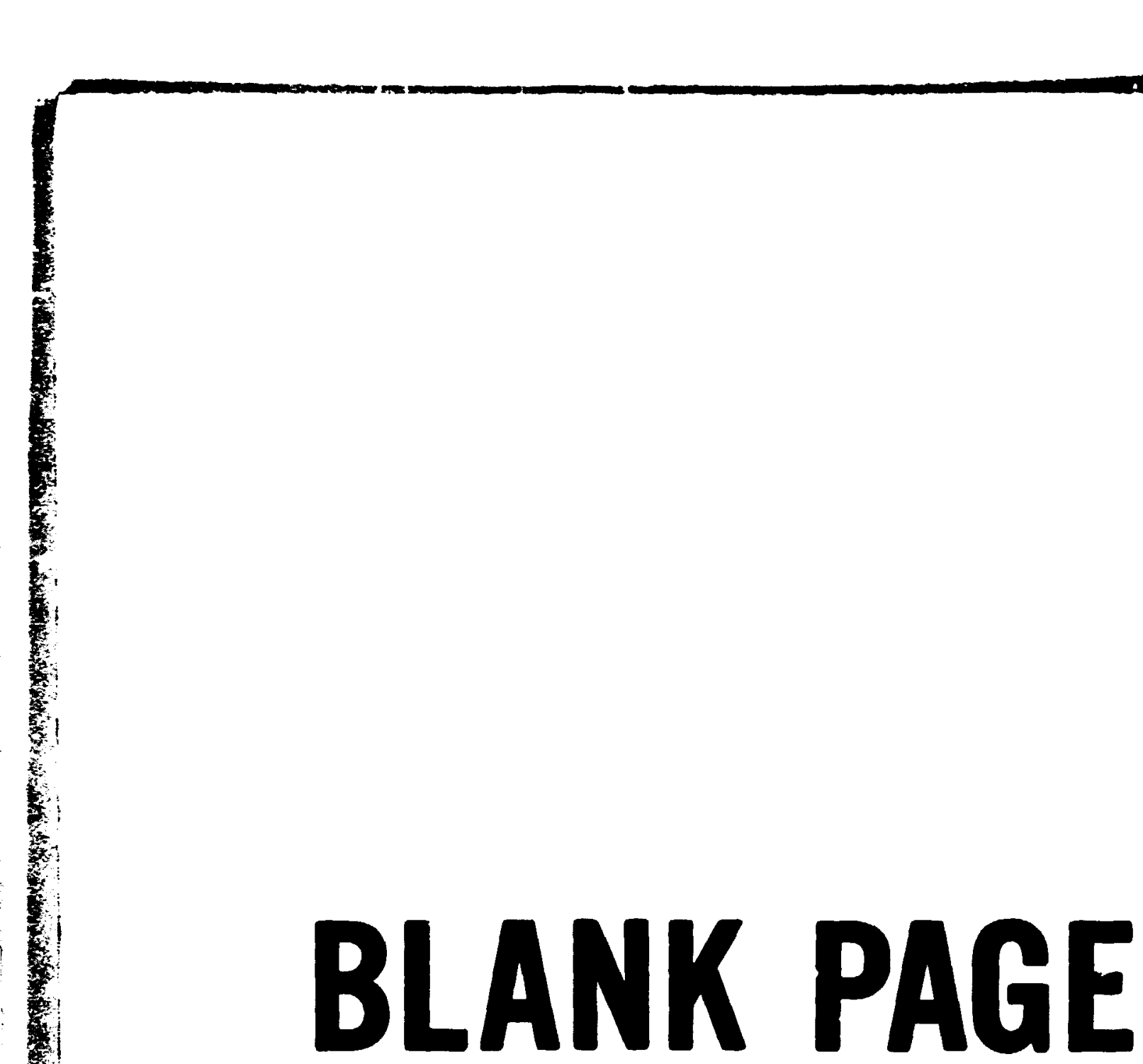




$$
\begin{aligned}
g^{-}(\gamma+k)+g^{-}(\gamma-k) & =\frac{88}{1-e^{i k a} e^{-k a}+(k-i k) l(1-a)} \\
& +\frac{e^{-i k a} e^{-k a}}{1-e^{-i k a} e^{-k a}+(k+i k) l(1-a)}
\end{aligned}
$$

This shows that the quantity $g^{-}(\gamma+k)+g^{-}(\gamma-k)$ is the sum of a complex quantity and its complex conjugate and hence is real. Therefore, all quantities in Eq. II.D.10 are real and so the self-energy is real. 